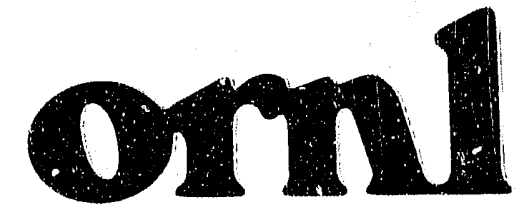

OAK RIDGE NATIONAL LABORATORY

MARTUR MARUSTA

\section{Time Optimal Trajectories for Mobile Robots with Two Independently Driven Wheels}

David B. Reister

François G. Pin 
This: report has been reproduced directly from the best availabie copy.

Avallable to DOE and DOE contractors from the Otfice of Scintific and Technical Information, P.O. BDx 62, Dak Aldge, TN 3783" prices available from (615) $570-10401$, FTS 826-8401.

Ayailable to the public from the National Technical Intormetion Service, U.S. Department of Commerce 5286 Port Foyal Fot., Springfield, VA 22161.

Thie report was preparad as an sccount of work sponsored by an agency of the United States Government. Melther the United States Government nor any agency thereof, nor any of their employese, makes any warranty, express or impilied, or assumes any legal liability or reaponsibility for the accuracy, complevisness; or usefulhess of eny information, apparafus, prodist, or procense disclosed, or represents that its use would not intringe privetely owned rights. Reference harein to any specific commercial product, procease, or asvivice by trade name, ti atemark, menufacturer, or otherwize, does not necesearily conatitute or imply its endarsement, recommendation, or favoring by the United States Government or any agency thereof. The views and opinions of authors expressed herein do not neres siarlly state or reflect those of the United Statea Government or any agency thisrat. 
Engineering Physics and Mathematics Division

\title{
TIME OPTIMAL TRAJECTORIES FOR MOBILE ROBOTS WITH TWO INDEPENDENTLY DRIVEN WHEELS
}

\author{
David B. Reister and François G. Pin
}

DATE PUBLISHED - March 1992

Research sponsored by the Engireering Research Program Office of Basic Energy Sciences U.S. Department of Energy

Prepared by the

OAK RIDGE NATIONAL LABORATORY

Oak Ridge, Tennessee 37831

managed by

MARTIN MARIETTA ENERGY SXSTEMS, INC.

for the

U.S. DEPARTMENT OF ENERGY

under contract DE-AC05-84OR21400 


\section{CONTENTS}

ABSTRACT . . . . . . . . . . . . . . . . . . . vii

1. INTRODUCTION

2. KINEMATIC EQUATIONS OF MOTION FOR THE PLATFORM . . 3

3. THE GENERAL FORM OF THE OPTIMAL CONTROLS . . . . . 5

4. PARAMETERIZATION OF THE BANG-BANG TRAJECTORIES . . 11

4.1 ANALYTICAL RELATIONSHIP BETWEEN THE SEGMENTS AND THE FINAL ORIENTATION . . . . . . . . . . . . . . . . . 13

4.2 CALCULATION OF THE DUAL VARIABLES . . . . . . . 14

5. BANG-BANG TRAJECTORIES . . . . . . . . . . . . 17

5.1 TRAJECTORIES TO A POINT . . . . . . . . . . . . . 17

5.2 TRAJECTORIES TO A CONFIGURATION . . . . . . . . 20

6. OPTIMAL TRAJECTORIES . . . . . . . . . . . . . 27

7. USE OF THE METHOD FOR ROBOT TRAJECTORY PLANNING , 35

8. CONCLUSIONS . . . . . . . . . . . . . . . . . 37

REFERENCES . . . . . . . . . . . . . . . . . . 39 


\section{LIST OF FIGURES}

$\underline{\text { Fig. }}$

1. The configuration of the platform is defined by the position $(x, y)$ and orientation $(\phi)$

2 Points that can be reached by trajectories with three switch times for a constant value of $T=\sqrt{10} \mathrm{sec}$

3 Curves showing the location of the furthest points in the first quadrant that can be reached by trajectories with three switch times for several values of $T\left(T^{2}=2,4,6,8\right.$, and 10$)$

4 Curve showing the ratio of $\tau^{m}$ and $\mathrm{T}$. The sets $\left(\tau^{m}, \mathrm{~T}\right)$ define the intersection of the first arcs with the $y$ axis

5 Cartesian paths of the robot for three-switch-time trajectories ending on the first arc defined by $\mathrm{T}^{2}=10$

6 Points that can be reached by trajectories with four switch times for a constant value of $T\left(T^{2}=10\right)$ when the final orientation is 0.8 radians

7 Points that can be reached by trajectories with four switch times for a constant value of $T\left(T^{2}=10\right)$ when the final orientation is 1.57 radians

8 Points that can be reached by trajectories with four switch times for a constant value of $T\left(T^{2}=10\right)$ when the final orientation is 3.14 radians

9 Curves showing the location of the furthest points that can be reached by trajectories with four switch times for several values of $\mathrm{T}\left(\mathrm{T}^{2}=2,4,6,8\right.$, and 10$)$ when the final orientation is 0.8 radians

10 Curves showing the location of points that can be reached by trajectories with four switch times for several values of $\mathrm{T}\left(\mathrm{T}^{2}=4,6,8\right.$, and 10) when the final orientation is 3.14 radians . . . . . . 25

11 Cartesian trajectories for paths with four switch times for a constant value of $T\left(T^{2}=10\right)$ when the final orientation is 0.8 radians

12. Cartesian trajectories for paths with four switch times for a constant value of $T\left(T^{2}=10\right)$ when the final orientation is 3.14 radians 
Fig.

13 Dual variables for a three-switch-time trajectory leading to the point $(0.66,4.03)$

14 Dual variables for a four-switch-time trajectory leading to the configuration $(0.20,3.43,0.80)$

15 Comparison of four-switch time trajectories of Type 22 and three-switch-time trajectories

16 Comparison of four-switch-time trajectories of Type 13 and three-switch-time trajectories

17 Comparison of five-switch-time trajectories of Type 23+- and four-switch-time trajectories of Type 22+-

18 Comparison of five-switch-time trajectories of Type $23+-$ and four-switch-time trajectories of Type 13+-

19 Comparison of five-switch-time trajectories of Type $23++$ and four-switch-time trajectories of Type $13++$

20 Comparison of five-switch-time trajectories of Type 14t- and four-switch-time trajectories of Type 13

21 Comparison of five-switch-time trajectories of Type $14++$ and four-switch-time trajectories of Type 13 
This paper addresses the problem of time-optimal motions for a mobile platform in a planar environment. The platform has two non-steerable independently driven wheels. The overall mission of the robot is expressed in terms of a sequence of via points at which the platform must be at rest in a given configuration (position and orientation). The objective is to plan time-optimal trajectories between these configurations assuming an unobstructed environment.

Using Pontryagin's maximum principle (PMP), we formally demonstrate that all time optimal motions of the platform for this problem occur for bang-bang controls on the wheels (at each instant, the acceleration on each wheel is either at its upper or lower limit). The PMP, however, only provides necessary conditions for time optimality. To find the time optimal robot trajectories, we first parameterize the bang-bang trajectories using the switch times on the wheels (the times at which the wheel accelerations change sign). With this parameterization, we can fully search the robot trajectory space and find the switch times that will produce particular paths to a desired final configuration of the platform. We show numerically that robot trajectories with three switch times (two on one wheel, one on the other) can reach any position, while trajectories with four switch times can reach any configuration. By numerical comparison with other trajectories involving similar or greater numbers of switch times, we then identify the sets of time-optimal trajectories. These are uniquely defined using ranges of the parameters, and consist of subsets of trajectories with three switch times for the problem when the final orientation of the robot is not specified, and four switch times when a full final configuration is specified. We conclude with a description of the use of the method for trajectory planning for one of our robots, and discuss some comparisons of sample time-optimal paths with minimum-length paths. 


\section{INTRODUCTION}

A variety of platform designs have been implemented for mobile robots. These can be classified in three major categories: 1 . omnidirectional platforms, that utilize steerable wheels (e.g., see robots in Brooks (1990), Arkin (1990), or Koren and Borenstein (1991)], roller-equipped wheels [e.g., see Blaisdell (1991)] or orthogonal wheel assemblies [e.g., see Killough and Pin (1990); $; 2$ car-like platforms that incorporate controlled steerable wheels on one axle and non-steerable wheels on another axle [e.g., see Vasseur, Pin, and Taylor (1991)]; and 3. skid-steer platforms that include two non-steerable independently driven wheels [e.g., see Giralt, Chatila, and Vaisset (1984), Kanayama and Hartman (1989), or Weisbin et al. (1989)]. This paper is concerned with skid-steer platforms. A typical mission for a mobile robot can be described by a sequence of via points a.t which the robot comes to rest in a given configuration (position and orientation) to perform a given task (manipulation, sensing, etc.). The problem considered in this paper is that of finding time-optimal motions of the robot in Cartesian space and the corresponding control trajectories that will move the robot from an initial configuration to a final configuration in an unobstructed environment.

The proposed approach to find the control trajectories that lead to time-optimal motions of the platform involves utilization of Pontryagin's maximum principle [Pontryagin et al. (1986)]. A variety of authors [Kahn and Roth (1971), Niv and Auslander (1984), Kim and Shin (1985), Weinreb and Bryson (1985), Nakamura and Hanafusa (1987), Bobrow (1988), Yamamoto and Mohri (1989)] have applied the maximum principle to the optimal motion planning of serial-link manipulators. For the time optimal motion of a manipulator with bounded control torques, the controls occur linearly in the Hamiltonian and the optimal values of the controls are determined by the dual variables. When its dual variable is not zero, the optimal control is bang-bang (the optimal control is at its upper limit for a positive dual variable and at its lower limit for a negative dual variable). When its dual variable is zero for a finite interval, the optimal control is singular and will be in the region between the bounds. An important issue is to determine when the optimal solution is bang-bang and when it is singular. A variety of authors (Ailon and Langholtz (1985), Wen (1986), Willigenburg (1990), and Chen and Desrochers (1990)] have addressed this issue and proven that there cannot be a finite time interval when the optimal control for a manipulator is singular for all the control torques. In other words, at least one of the controls is always bang-bang. Further work by Geering, et al. (1986) led to the determinatic $n$ of the parameter values for singular solutions for three types of manipulators: cylindrical, spherical, and two link planar. It has been demoustrated [Osipov and Formal'skii (1990), Formal'skii and Osipov (1090)] that the singular solution for the cylindrical case is not optimal. As discussed in the next section, the kinematics of a two-wheeled robot differ significantly frum that of a serial-link manipulator and, to our knowledge, the time optimal trajectories for a two-wheeled robot have never been found. 


\section{INTRODIICTION}

In the following sections, we present the equations of motion for a skid-steer type of platform moving on a flat, horizontal plane. We then use the maximum principle to derive the conditions for time-optimal motions of the platform and demcristrate that the optimal controls are always bang-bang. Using this result, we show that, for a system with bounded wheel accelerations, control trajectories with three switch times (times at which one of the wheel's acceleration changes sign) allow the robot to reach any point in Cartesian space while with four switch times, the robot can reach any configuration. We then show numerically that these paths are time-optimai. 


\title{
2. KINEMATIC EQUATIONS OF MOTION FOR THE PLATFORM
}

\begin{abstract}
A skid-steer type of platform must satisfy nonholonomic constraints and cannot follow an arbitrary path through configuration space. In this section, we develop a kinematic model of the platform, assuming that the wheels do not slip and that the wheel accelerations can instantaneously switch from their upper limit to their lower limit. The configuration of the platform is described by three coordinates: the Cartesian coordinates, $x$ and $y$, of the midpoint of the wheel axle with respect to an absolute reference frame, and the orientation, $\phi$, of the platform main axis with respect to the reference frame $x$ axis (see Fig. 1). The joint variables are the wheels' translational displacements, denoted by $\vartheta_{R}$ and $\theta_{L}$, (representing the angular rotation times the radius of the right and left wheels), and are measured in meters. The wheel velocities are denoted by $\omega_{R}$ and $\omega_{L}$ and are measured in meters per second. The control variables are the right and lefi wheel's translational accelerations $\left(u_{R}\right.$ and $\left.u_{L}\right)$. The kinematic model links the Cartesian variables to the control variables through the wheel velocities:
\end{abstract}

$$
\begin{gathered}
\dot{\phi}=\left(\omega_{R}-\omega_{L}\right) / D \\
\dot{x}=\left(\omega_{R}+\omega_{L}\right) \cos (\phi) / 2 \\
\dot{y}=\left(\omega_{R}+\omega_{L}\right) \sin (\phi) / 2 \\
\dot{\omega}_{R}=u_{R} \\
\dot{\omega}_{L}=u_{L} \\
\dot{\theta}_{R}=\omega_{R} \\
\dot{\theta}_{L}=\omega_{L}
\end{gathered}
$$

where $D$ is the distance between the centers of the wheels.

Given a trajectory for the control variables, Eqs. (1) through (7) can be integrated to determine the Cartesian variables and the joint variables. Contrary to what is typically the case for serial link manipulators, the time sequence of the controls is necessary to determine the final position of is two-wheeled platform. For example, if both wheels rotate together and move a meter, the platform will move straight forward a meter, while if the right wheel moves a meter first and then the left wheel r.coves a meter, the platform will move to the left to a point less than a meter away. Although the final values of the joint variables and the final platform orientation are the same for the twr maneuvers, the final values ior the platform position are not the same. 


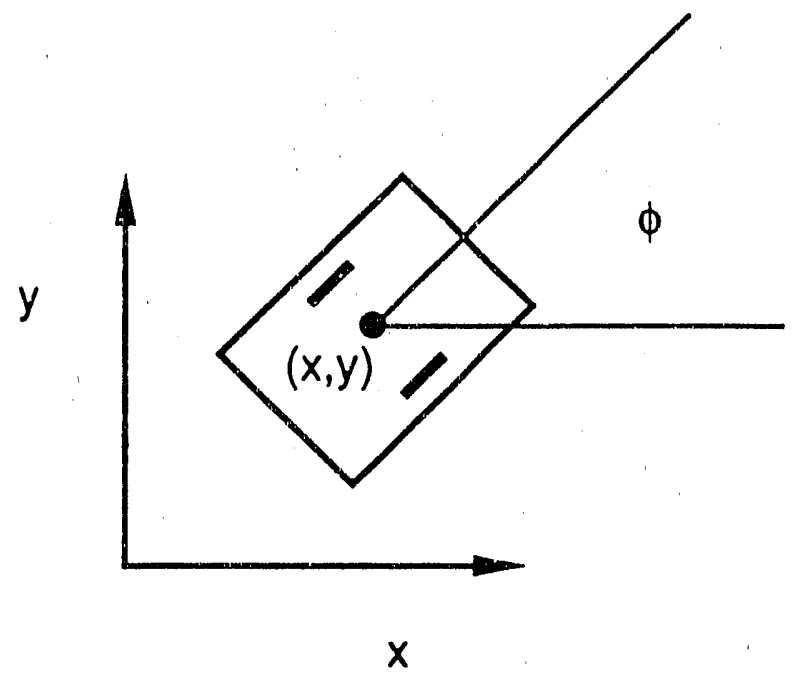

Fig. 1. The conflguration of the platform is deflned by the position $(x, y)$ and orientation $(\phi)$. 


\section{THE GENERAL FORM OF THE OPTIMAL CONTROLS}

Since its discovery in 1956, the Pontryagin maximum principle [Pontryagin (1986)] has been used to solve a wide variety of optimization problems. In this section we utilize the inaximum principle to derive the conditions for time optimality of the platform motion between the initial and final configurations. For our problem, we can define five state variables: $x_{1}=x, x_{2}=y, x_{3}=\phi, x_{4}=\omega_{R}$, and $x_{5}=\omega_{L}$ and rewrite the equations of motion (1) to (5) as:

$$
\begin{gathered}
\dot{x}_{1}=f_{1}(x, u)=\left(x_{4}+x_{5}\right) \cos x_{3} / 2 \\
\dot{x}_{2}=f_{2}(x, u)=\left(x_{4}+x_{5}\right) \sin x_{3} / 2 \\
\dot{x}_{3}=f_{3}(x, u)=\left(x_{4}-x_{5}\right) / D \\
\dot{x}_{4}=f_{4}(x, u)=u_{1} \\
\dot{x}_{5}=f_{5}(x, u)=u_{2}
\end{gathered}
$$

where the two control variables are: $u_{1}=u_{R}$ and $u_{2}=u_{L}$.

In vector notation, the equations of motion for the state vector $x$ are:

$$
\dot{x}=f(x, u),
$$

and the optimization problem is to find a control vector $[u]$ that will move the system from the initial state $x^{0}$ to the final state $x^{1}$ while minimizing an objective functional. For our case, the goal is to minimize the transition time, assuming that the wheel accelerations are bounded: $\left|u_{1}\right| \leq a_{\max }$ and $\left|u_{2}\right| \leq a_{\max }$.

Pontryagin introduces a system of dual variables $[\psi]$ that satisfy:

$$
\dot{\psi}_{i}=-\sum_{j=1}^{n} \frac{\partial f_{j}(x, u)}{\partial x_{i}} \psi_{j}, \quad i=1,2, \ldots, n
$$

with initial conditions:

$$
\psi_{i}\left(t_{0}\right)=\lambda_{i} \quad i=1,2, \ldots, n,
$$

where, in our problem, $n=5$.

If we choose an admissible control and have the solution of Eq. (13), Eqs. (14) are linear and homogeneous and have a unique solution for given initial conditions. If the equations of motion and the dual variables are combined into a single Hamiltonian $H$ : 


$$
H(\psi, x, u))=\sum_{j=1}^{n} \psi_{j} f_{j}\left((x, w)^{\prime},\right.
$$

then the PMIP states that [see Theorem 2 in Pontryagin (1986)], if $u$ is an admissible control that transfers the phase point from the initial statte $x\left(t_{0}\right)=x^{0}$ to the goal state $x^{1}=x\left(t_{11}\right)$ at some tinne $t_{1}$, then $u(t)$ and the trajectory $x(t)$ are time-optimal if there exists a non-zexo contimous vector function $\left.\left(\psi_{1}(t), \ldots . \psi_{n}(t)\right)\right)$ defined by Eq. (14)), and sucki that $u(t)$ maximizes the function $H$ for all $t, t_{0} \leq t \leq t_{1}$.

For our mobile platform problem desined by Eqs. (8) through (12), the Hlamiltonis fur fiction $H$ is:

$$
H(\psi, x, w)=\psi_{11} f_{1}+\psi_{2} f_{2}+\psi_{3} f_{3}+\psi_{4} w_{1}+\psi_{5}, w_{2}
$$

where the functions $f_{i}(x, w)$ are defined by Eqs. (8) to (12). The equations for the duall variables are:

$$
\begin{gathered}
\dot{\psi}_{1}=0 \\
\dot{\psi}_{2}=0 \\
\dot{\psi}_{3}=g\left(x_{3}\right)\left(x_{4}+x_{5}\right) \\
\dot{\psi}_{4}=-g^{\prime}\left(x_{3}\right)-\psi_{3} / D \\
\dot{\psi}_{5}=-g^{\prime}\left(x_{3}\right)+\psi_{3} / D
\end{gathered}
$$

where $g\left(x_{3}\right)$ and $g^{\prime}\left(x_{3}\right)$ are defined by:

$$
\begin{gathered}
g\left(x_{3}\right)=\left(\psi_{1} \sin x_{3}-\psi_{2} \cos x_{3}\right) / 2 \\
g^{\prime}\left(x_{3}\right)=\frac{d g\left(x_{3}\right)}{d x_{3}}=\left(\psi_{1} \cos x_{3}+\psi_{2} \sin x_{3}\right) / 2
\end{gathered}
$$

The platform moves from an arbitrary initial configuration to an arbitrary final configuration. We can choose the coordinate system such that the initial position is $(0,0)$ and the initial orientation is $\phi=0$. The boundary conditions corresponding to the robot being at rest at the initial state $\left.x^{0}=x\left(t_{0}\right)\right)$ and final state $x^{1}=x\left(t_{1}\right)$, are

$$
\begin{aligned}
& x\left(t_{01}\right)=(0,0,0,0,0) \\
& x\left(t_{1}\right)=(x, y, \phi, 0,0)
\end{aligned}
$$


When the final state is fully specified (position and orientation are given), the finall values of the duall variables are arbitrary. When the finall state is not fullly specified, the transversality condition determines the final values of the duall variables [see Thenrern 3 in Pontryagin (1986)]. For example, when the final state is a posittion (and the final orientation is not specified), the final value of the third duali variable $(1 \%$ is $)$ must be zero.

The optimal values for the control variables: ( $u_{1}$ and $u_{2}$ ) are those that maximize the Hamilitonian. From Eq. (17), it is clear that when the dual variables $\psi_{4}$ and $\psi_{5}$ are not zero, the optimal control is bang-bang; when $\psi_{4}$ is positive, $u_{i}=a_{\max }$ and when $\psi_{4}$ is negative, $u_{1}=-a_{\max }$, and similarly for $\psi_{5}$ and $w_{2}$. If $\psi_{4} \psi_{4}$ or $\psi_{5}$, is zero for a finite time interval, the optimal control becomess singular. In the remaining of this section we will prove that all possible optimal solutions of the system are baing-bang.

\section{Theorem}

All opuimal control solutions for the system defined by E'qs. (8) through (12), (25) and (26) are bang-bang.

\section{Proof}

From Eq. (17), we know that when the dual variables $\psi_{4}$ and $\psi_{5}$ are not zero, the optimal control is bang-bang; and when $\psi_{4}$ or $\psi_{5}$ is zeris for a finite interval, the optimal control is singular. To prove the thenrem, we vrill investigate all the singular solutions and show that they are either impossible or bang-bang.

When the optimal control is singular, $\psi_{4}$ (or $\psi_{5}$ ) is zero for a ?nite interva". If $\psi_{4}$ is zero for a fimite time interval, $\left[t_{2}, t_{3}\right]$, all of its derivatives are also zero on the interval and Eq. (21) yields:

$$
\psi_{3}=-D_{g}{ }^{\prime}\left(x_{3}\right)
$$

The time derivative of Eq. (27) implies:

$$
\dot{\psi}_{3}=-D \frac{d g^{\prime}\left(x_{3}\right)}{d x_{3}} \dot{x}_{3}=g\left(x_{3}\right)\left(x_{4}-x_{5}\right)
$$

where we have used the fact that $\frac{d g^{\prime}\left(x_{3}\right)}{d x_{3}}=-g$.

Since the right sides of Eqs. (20) and (28) must be equal, we have:

$$
g\left(x_{3}\right) x_{5}=0
$$

Both $g\left(x_{3}\right)$ and $x_{5}$ are continuous functions. If there is a point in $\left[t_{2}, t_{3}\right]$ where $x_{5}$ is not equal to zero, then there is a finite interval where $x_{5}$ is not equal to zero and on this subintervall $g\left(x_{3}\right)$ is zero. Similarly, if there is a point in $\left[t_{2}, t_{3}\right]$ where $g\left(x_{3}\right)$ 


\section{THE GENEITAL FORM OF THE OPTIMAL CONTROLS}

is not equall to zero, then there is 2 subunterval where $x_{5}$ is zero. Thus, we will consider two general cases:

Case 1. $\quad g\left(x_{3}\right)=0$

Case 2. $\quad x_{5}=0$

To simplify the rotation, we will continue to label the subintervals of $\left[t_{2}, t_{3}\right]$ where Eqs. (30) or (31) are valid $\left[t_{2}, t_{3}\right]$.

Case 1 can be subdivided into four subcases:

Case 1.1. $\quad \psi_{1} \neq 0, \quad \psi_{2} \neq 0$

Case 1.2. $\quad \psi_{1}=0 \quad \psi_{2} \neq 0$.

Case 1.3. $\quad \psi_{1} \neq 0 \quad \psi_{2}=0$

Case 1.4. $\quad \dot{\psi}_{1}=\psi_{2}=0$

For the first three subcases, $x_{3}$ is a constant on the interval $\left[t_{2}, t_{3}\right]$ :

Case 1.1. $\quad$ tan $x_{3}=\psi_{2} / \psi_{1}$

Case 1.2. $\quad \cos x_{3}=0$

Case 1.3. $\quad \sin x_{3}=0$

\section{Consider Cases 1.1, 1.2, and 1.3}

Since $\psi_{1}$ and $\psi_{2}$ are constant (from Eqs. (18) and (19)) and $g\left(x_{3}\right)=0, x_{3}$ is a constant and $\dot{x}_{3}=0$ on the finite interval $\left[t_{2}, t_{3}\right]$. Thus, these cases correspond to the robot moving along a straight line over a finite interval of time, (the angle $\phi=x_{3}$ given by Eqs. (32), (33), or (34)') and Eq. (10) requires that $x_{4}=x_{5}$, i.e., that the controls on both wheels be equal, $u_{1}=\dot{x}_{4}=\dot{x}_{5}=u_{2}$, over the interval $\left[t_{2}, t_{3}\right]$. Now, since $g=0, \dot{\psi}_{3}=0$ (using Eq. (20)), and $\psi_{3}$ is a constant. Since $g^{\prime}$ is also a constant $\left(\psi_{1}, \psi_{2}\right.$, and $x_{3}$ are constants in Eq. $\left.(24)\right)$, $\dot{\psi}_{5}$ is constant (from Eq. (22)).

If $\dot{\psi}_{5}$ is a non-zero constant, then $\psi_{5}$ varies linearly, and the optimal controk $u_{2}$ and $u_{1}$ are equal and bang-bang over the interval $\left[t_{2}, t_{3}\right]$. If $\dot{\psi}_{3}$ is zero, then Eq. (22) yields $\psi_{3}=D g^{\prime}\left(x_{3}\right)$ which, with Eq. (27), implies $\psi_{3}=g^{\prime}\left(x_{3}\right)=0$. If both $g=0$ and $g^{\prime}=0$, then $\psi_{1}=\psi_{2}=0$. This violates the assumptions in Eqs. (32), (33), or 
(34) and consequently $\psi_{5}=0$ is not a valid solution for these cases. Thus, for cases $1.1,1.2$, wnd 1.3, the only admissible controls are bang-bang.

\section{Consider Case 1.4}

If $\psi_{1}=\psi_{2}=0$ over the interval $\left[t_{2}, t_{3}\right]$, they are also zero over the entire trajectory (from Eqs. (18) and (19)). Thus $g=g^{\prime}=0$ and $\dot{\psi}_{3}=0$ (from Eq. (20)) over the entire trajectory. Furthermore, since $\psi_{3}$ is a constant and $\psi_{3}=\hat{U}$ (from Eq. (27)i) on the interval $\left[t_{2}, t_{3}\right], \psi_{3}=0$ over the entire trajectory. Consequently, $\dot{\psi}_{5}=0$ from Eq. (22), requiring the dual variable $\psi_{5}$ to be constant over the entire trajectory. This is not an admissible case for our problem since the corresponding extremal controls $u_{2}$ would not change sign over the entire trajectory, leading to a linearly increasing or decreasing wheel velocity and making Eq. (26) impossible to satisfy. Thus case 1.4 does not lead to admissible controls for our problem.

\section{Consider Case 2}

If $x_{5}=0$ over a finite time interval $\left[t_{2}, t_{3}\right]$, then the control $u_{2}=\dot{x}_{5}=0$ over the interval, and consequenuly its dual variable $\psi_{5}$ and its derivative $\psi_{5}$ must be zero over the entire finite interval. Since $\psi_{4}=\dot{\psi}_{4}=0$, Eqs. (21) and (22) require $\psi_{3}=0$ and $g^{\prime}\left(x_{3}\right)=0$ over the entire interval, and consequently $\dot{\psi}_{3}=0$. Equation (20) thus requires either that $x_{4}=x_{5}=0$, which is an inadmissible case (since, from Eqs. (8) through (12), no motion of the robot would take plact over a finite time interval during the trajectory, which consequently can not be time optimal), or that $g\left(x_{3}\right)=0$ over the finite time interval. If both $g$ and $g^{\prime}$ are zero over the finite time interval, then $\psi_{1}=\psi_{2}=0$. Since $\psi_{1}$ and $\psi_{2}$ are constant over the entire trajectory (from Eqs. (18) and (19)), they must be zero over the entire trajectory and Eq. (23), (24), and (20) lead to $g=g^{\prime}=\dot{\psi}_{3}=0$ over the entire trajectory. Consequently, since $\psi_{3}=0$ over the finite time interval and $\dot{\psi}_{3}=0$ over the entire trajectory, $\psi_{3}=0$ and $\dot{\psi}_{4}=\dot{\psi}_{5}=0$ (from Eqs. (21) and (22)) over the entire trajectory. Therefore, since $\psi_{4}=0$ and $\psi_{5}=0$ over the finite interval, they also are zero on the entire trajectory. Thus this case is not admissible since all dual variables $\psi_{i}$ are zero over the entire trajectory.

Since the equations for $\psi_{4}$ and $\psi_{5}$ have the same structure, similar arguments demonstrate that singular solutions corresponding to the dual variable $\psi / 5$ being zero over a finite time interval lead to optimal controls that are bang-bang, or are inadmissible.

If $\psi_{4}=\psi_{5}=0$ over a fnite interval $\left[t_{2}, t_{3}\right]$, then $\dot{\psi}_{4}=\dot{\psi}_{5}=0$ over the interval and Eqs. (21) and (22) require that $g^{\prime}\left(x_{3}\right)=\psi_{3}=0$ over the interval. Since $\dot{\psi}_{1}$ and $\psi_{2}$ are constant, $g^{\prime}\left(x_{3}\right)=0$ implies that $x_{3}$ is constant over the entire interval. Thus $\dot{x}_{3}=0$ and, from Eq. (10), $x_{4}=x_{5}$ over the entire interval. On the other hand, $\psi_{3}=0$ over the entire interval implies $\dot{\psi}_{3}=0$, and $\mathrm{Eq} .(20)$ requires either $x_{4}=-x_{5}$ which, with the conclusion of the previous sentence, leads to $x_{4}=x_{5}=0$ 


\section{THE GENERAL FORM OF THE OPTIMAL CONTROLS}

(i.e., the robot does not move during, the entire interval), which is not an admissible solution; or $g\left(x_{3}\right)=0$, which has been treated as Case 1 above and shown to lead to bang-bang or inadmissible controls. Thus, all optimal control solutions for the system dcfined by Eqs. (8): through (12), (25), and (26) are bang-bang.

A similar result was recently outlined by Jacobs, Laumond and Rege (1991), however, without consideration given to cases $1.2,1.3,1.4$, and $\psi_{4}=\psi_{5}=0$ in the demonstration. 


\section{PARAMETERIZATION OF THE BANG-BANG TRAJECTORIES}

The Pontryagin maximum principle converts the problem of time-optimal path planning from a problem involving trajectories in state space and time to a static optimization problem in parameter space. Two sets of parameters are available: the initial conditions for the dual variables $\left[\lambda_{k}\right]$ and the switch times. Using either set of parameters, nonlinear search techniques can be used to determine bang-bang control trajectories that move the robot from the initial state to the final state. In the remainder of this paper, we will use the switch times to parameterize the control trajectories and the corresponding robots paths. The PMP provides necessary (but not sufficierit) conditions for an optimal trajectory. In the following sections, we will indeed find trajectories that satisfy the necessary conditions but are not timeoptimal. To show that a bang-bang trajectory is time-optimal, we must show that it satisfies the necessary conditions and that the corresponding robot path is faster than all alternative paths.

To show that a bang-bang trajectory satisfies the necessary conditions, we will numerically integrate the controls of the wheels to calculate the Cartesian path of the robot and some auxiliary variables, use the auxiliary variables to calculate the initial conditions for the dual variables, numerically integrate to calculate the dual variables, and verify that the necessary conditions are satisfied by showing that the dual variables are consistent with the bang-bang controls. In this sertion, we parameterize the trajectories, define the auxiliary variables, and show how to calculate the initial conditions for the dual variables. In the next two sections, we will explore the space of bang-bang trajectories and uniquely identify those that are time-optimal.

We have proven that the optimal controls are bang-bang. Thus, each wheel is always either accelerating or decelerating at the maximum rate $\left(a_{\max }\right)$, and the wheel velocity trajectories consist of successive segments of linearly increasing or decreasing velocity. The wheel acceleration changes sign at a switch time. We can characterize a wheel control trajectory by its number of switch times, and a robot path by its total number of switch times and their distribution on either of the two wheels. We will find that, for given total trajectory times, a specific set of robot paths with a small number of switch times reach farther (and therefore are faster) than all paths with a larger number of switch times. At the lower bound, the path with the smallest number of switch times has two (one for each wheel). However, inere are only two paths with two switch times: a translation straight forward and a pure rotation. On the other hand, we know that the minimum length paths for a skid-steer platform consist of sequences of translations and rotations. A rotation followed by a translation can reach ary position and requires five switch times, while a path consisting of a rotation, a translation, and a rotation can reach any configuration and has eight switch times. In the next section, we will show that the robot can rearh any position in the plane using specific paths involving a total of three switch times (one on one wheel, two on the other), and does so faster than 
with a greater number of switch times. Similarly, we will show that, using specific paths with four switch times, the robot can reach any configuration faster than with paths involving a greater number of switch times.

First, we derive an analytical expression for the displacement of a single wheel with four switch times. By adjusting parameters, the expression will yield the displacement for motions with one, two, or three switch times. We assume that the initial value for the whel displacement $(\theta)$ is zero. Since the initial and final values for the wheel velocity are zero, the wheel will accelerate during half of the trajectory time, and it will decelerate during the other half. We will denote by $T$ half of the total trajectory time.

A control trajectory with four swritch times has five time segments denoted by $\left(\tau_{i}\right)$. For two wheels, we can define ten sigments; our notation for the right segments will be $\left(\tau_{1}, \tau_{3}, \tau_{5}, \tau_{7}, \tau_{9}\right)$, while the left segments will be $\left(\tau_{2}, \tau_{4}, \tau_{6}, \tau_{8}, \tau_{10}\right)$. Let $u_{R}$ be the initial acceleration on the right wheel. The control trajectory for the right wheel will be: $u_{R}$ for $\left[0, \tau_{1}\right],-u_{R}$ for $\left[\tau_{1}, \tau_{1}+\tau_{3}\right], u_{R}$ for $\left[\tau_{1}+\tau_{3}, \tau_{1}+\tau_{3}+\tau_{5}\right],-u_{R}$ for $\left[\tau_{1}+\tau_{3}+\tau_{5}, \tau_{1}+\tau_{3}+\tau_{5}+\tau_{7}\right]$, and $u_{R}$ for $\left[\tau_{1}+\tau_{3}+\tau_{5}+\tau_{7}, 2 T\right]$. Since the wheel will accelerate during half of the trajectory, and it will decelerate during the other half:

$$
\begin{gathered}
\tau_{1}+\tau_{5}+\tau_{9}=\mathrm{T} \\
\tau_{3}+\tau_{7}=\mathrm{T}
\end{gathered}
$$

Integrating Eqs. (4) and (6), we can calculate the final value of the right wheel displacement for a four-switch-time trajectory of that wheel:

$$
\theta_{R}(2 \mathrm{~T})=u_{R}\left[-2 \tau_{3} \tau_{5}+2\left(\tau_{1}+\tau_{5}\right) \mathrm{T}-\mathrm{T}^{2}\right]
$$

Equation (38) relates the wheel displacement to the first three segments. Using Eqs. (36) and (37), we can relate the wheel displacement to the last three segments:

$$
\theta_{R}(2 \mathrm{~T})=u_{R}\left[2 \tau_{5} \tau_{7}-2\left(\tau_{5}+\tau_{9}\right) \mathrm{T}+\mathrm{T}^{2}\right]
$$

The similar expressions for the displacement of the left wheel are:

$$
\begin{gathered}
\tau_{2}+\tau_{6}+\tau_{10}=\mathrm{T} \\
\tau_{4}+\tau_{8}=\mathrm{T} \\
\theta_{L}(2 \mathrm{~T})=u_{L}\left[-2 \tau_{4} \tau_{6}+2\left(\tau_{2}+\tau_{6}\right) \mathrm{T}-\mathrm{T}^{2}\right] \\
\theta_{L}(2 \mathrm{~T})=u_{L}\left[2 \tau_{6} \tau_{8}-2\left(\tau_{6}+\tau_{10}\right) \mathrm{T}+\mathrm{T}^{2}\right]
\end{gathered}
$$

with $u_{L}$ representing the initial acceleration of the left wheel. 


\subsection{ANALYTICAL RELATIONSHIP BETWEEN THE SEGMENTS AND THE FINAL ORIENTATION}

Although the equations of motion for the Cartesian position variables $(x, y)$ do not have general analytical solutions, a solution for the Cartesian orientation variable $\phi$ can be derived analytically. Using Eqs. (6) and (7), Eq. (1) may be written:

$$
\dot{\phi}=\left(\dot{\theta}_{R}-\dot{\theta}_{L}\right) / D
$$

Since the initial conditions for the wheel displacement joint variables are zero, Eq. (44) can be integrated to yield:

$$
\phi=\phi_{0}+\left(\theta_{R}-\theta_{L}\right) / D
$$

where $\phi_{0}$ is the initial value of the robot's orientation.

Equations (38), (39), (42), and (43) relate the segment lengths to the wheels' displacement, while Eq. (45) relates the wheels' displacement to the change in orientation. Given segment lengths for both wheels, the final change in orientation can be calculated. Alternatively, given the desired final orientation of the robot, Eq. (45) provides a constraint on the segment lengths.

Consider a robot path involving three switch times; one on the right wheel and two on the left wheel (the segment lengths are: $\tau_{1}=\tau_{3}=\tau_{4}=\mathrm{T}$, and $\left.\tau_{5}=\tau_{7}=\tau_{8}=\tau_{9}=\tau_{10}=0\right)$. Since $\tau_{2}+\tau_{6}=T$, this path is defined by two parameters $\left(\tau_{2}\right.$ and $T$ ). Using Eqs. (39) and (43), the final wheel displacements are:

$$
\begin{gathered}
\theta_{R}=u_{R} \mathrm{~T}^{2} \\
\theta_{L}=u_{L}\left[T^{2}-2 \tau_{6} \mathrm{~T}\right]
\end{gathered}
$$

Since the initial value of the robot's orientation is zero, the final change in orientation is:

$$
\phi D=u_{R} \mathrm{~T}^{2}-u_{L}\left[\mathrm{~T}^{2}-2 \tau_{6} \mathrm{~T}\right]
$$

If $u_{R}=u_{L}=u$, the final orientation is:

$$
\phi D=2 u \tau_{6} T
$$

while if $u_{R}=-u_{L}=u$, the final orientation is:

$$
\phi D=2 u \tau_{2} T
$$

If the final orientation is specified and is positive (negative), then $u$ must be positive (negative). Moreover, if a final orientation is specified for a three-switch-times path, Eqs. (49) and (50) may not have acceptable solutions for small values of $T$, since $0 \leq \tau_{i} \leq \mathrm{T}$. On the other hand, if no constraint on the final orientation is specified for a path, Eqs. (49) and (50) show that as $\tau_{2}$ or $\tau_{6}$ varies from zero to $T$, the 
change in orientation varies continuously from zero (the motion is a translation straight forward) to $2 u \mathrm{~T}^{2} / D$ (the motion is a pure rotation).

\subsection{CALCULATION OF THE DUAL VARIABLES}

To verify that a bang-bang trajectory satisfies the necessary conditions, we must calculate the dual variables and show that they are consistent with the control paths (i.e., that $u_{1}$ is positive (negative) when $\psi_{4}$ is positive (negative), etc.]. The dual variables satisfy Eqs. (18) to (22), with initial conditions given by Eq. (15). Since this set of equations is linear and homogeneous, it has a unique solution for any set of initial conditions $\left(\lambda_{i}\right)$. Thus, the initial conditions determine the trajectories of the dual variables. From Eqs. (18) and (19), the first two dual variables are constants $\left(\psi_{1}=\lambda_{1}\right.$ and $\left.\psi_{2}=\lambda_{2}\right)$. Using Eqs. (8), (9), (20), and (23), the third dual variable satisfies:

$$
\dot{\psi}_{3}=\psi_{1} \dot{x}_{2}-\psi_{2} \dot{x}_{1}
$$

Since the initial position is $(0,0)$, Eq. (51) can be integrated:

$$
\psi_{3}=\lambda_{1} x_{2}-\lambda_{2} x_{1}+\lambda_{3}
$$

The fourth and fifth clual variables satisfy Eqs. (21) and (22). The right sides of these equations depend on the first three state variables. To integrate the equations, we define four auxiliary variables $\left(z_{i}\right)$ by:

$$
\begin{array}{cr}
\dot{z}_{1}=\sin \left(x_{3}\right) & z_{1}(0)=0 \\
\dot{z}_{2}=\cos \left(x_{3}\right) & z_{2}(0)=0 \\
\dot{z}_{3}=x_{1} & z_{3}(0)=0 \\
\dot{z}_{4}=x_{2} & z_{4}(0)=0
\end{array}
$$

Using the auxiliary variables, the solutions for the fourth and fifth dual variables are:

$$
\begin{gathered}
\psi_{4}=-\lambda_{1}\left(z_{2} / 2+z_{4} / D\right)+\lambda_{2}\left(z_{3} / D-z_{1} / 2\right)-\lambda_{3}(-/ D)+\lambda_{4} \\
\psi_{5}=\lambda_{1}\left(z_{4} / D-z_{2} / 2\right)-\lambda_{2}\left(z_{1} / 2+z_{3} / D\right)+\lambda_{3}(\tau / D)+\lambda_{5}
\end{gathered}
$$

Since the switch times of a trajectory correspond to times when either $\psi_{4}$ or $\psi_{5}$ changes sign, one of the dual variables must be zero at the switch time. Thus, the initial conditions for the dual variables can be determined by solving the matrix equation: $A \lambda=0$, where $\lambda^{\mathrm{T}}=\left(\lambda_{1}, \ldots, \lambda_{5}\right)$ and the rows of the matrix $A$ are defined by either Eqs. (57) or (58) applied at the switch times. If the switch time 
is for the right wheel, $\psi_{4}$ is equal to zero and Eq. (57) defines a row of $A$. If the switch time is for the left wheel, $\psi_{5}$ is equal to zero and Eq. (58) defines a row of $A$. It is interesting to note here that, in the general case, a control path with $n$ switch times will produce a matrix witl $n$ rows. For the vector $\lambda$ to be nonzero, the rank of $A$ must be four or less. In other words, if a control path has less than five switch times, a nonzero solution is possible. If the control path has more than four switch times, the columns of the $A$ matrix must be linearly dependent. Thus, in the next section, we will first explore the space of robot paths involving four switch times in order to investigate general solutions of our problem when the final configuration (position and orientation) is specified. A particular case occurs when the final state is a position (and the final orientation is not specified). In that case, the transversality condition [see Pontryagin (1986) Theorem 3, page 50] requires that the final value of the third dual variable be zero and $\mathrm{Eq}$. (52) defines a row of $A$. Thus, when the final state is a position, a path with three switch times would produce a four row matrix. In the next section, we will therefore explore the space of robot paths involving three switch times to investigate general solutions of the problem when the final orientation is not specified. 


\section{BANG-BANG TRAJECTORIES}

Our objective is to move from an arbitrary initial configuration to an arbitrary final configuration in minimum time. Each configuration is described by three coordinates $(x, y, \phi)$, where $(x, y)$ is the location of the midpoint betweer the two wheels and $\phi$ is the orientation of the robot. We can choose the coordinate systern such that the initial configuration is $(0,0,0)$. The initial and final values for the wheel velocities $\left(\omega_{R}\right.$ and $\left.\omega_{L}\right)$ are zero.

We will first explore the spaces of paths with three and four switch times. With the parameterization described in the previous section, we can vary the available parameters over the entire range, and for each set of parameter values, we can numerically integrate the equations of motion using a fourth-order Runge-Kutta rnethod [Press (1988)] to determine the Cartesian path of the platform and its final position and orientation. To produce the example figures in this section, we have used $a_{\max }=0.5$ meters $/$ second $^{2}$ for the acceleration bound on the wheels, and $D=0.76$ meters for the wheels' spacing.

\subsection{TRAJECTORIES TO A POINT}

When the goal is to reach a given point with no specified orientation, the problem is symmetricai and can be solved considering only the first quadrant. If any point in the first quadrant can be reached, symmetrical points in the other three quadrants can be reached by changing the sign of the wheels' controls, i.e., changing the initial direction of motion (symmetry about the $y$ axis) or by exchanging the trajectories for the two wheels (symmetry about the $x$ axis). Given the symmetry conditions, we are exploring three switch times trajectories involving one switch time for the right wheel and two for the left. wheel. As mentioned previously, the three switch time trajectories with no specified final orientation can be parameterized using two parameters and can be of two types, which we refer to as: Type ++ (on the initial segment, $\left.u_{R}=u_{L}=u\right)$ and Type $+-\left(u_{R}=-u_{L}=u\right)$.

Figure 2 shows the final locations of the platform for the two types of solutions when one of the parameters, the half time $T$ of the trajectory, is kept at a fixed value, $T=\sqrt{10}$ sec. The Type ++ trajectories initiate with a translation and end with a rotation, while the Type +- trajectories initiate with a rotation and end with a translation. As the parameter $\left(\tau=\tau_{2}\right.$ or $\left.\tau_{6}\right)$ increases from zero to $T$, the final positions of the robot for both types of trajectories describe a curve from the point $(5,0)$ to the origin, with the motion varying from a pure translation reaching the point $(5,0)$ for $\tau=0$, to a pure rotation of the robot at the origin for $\tau=T$.

Considering the first quadrant and all symmetries involved in the problem, it is clear that the trajectories of Type +- that end on the arc $A B$, which we thereafter call "first arc," can reach points further away from the origin than the other trajectories of Type +- and all trajectories of the Type ++. In Fig. 3, the final platform locations which correspond to first arcs are plotted fo: several values of $\mathrm{T}$. Since for pure translation, the platform moves a distance $x$ along the $x$-axis: 


$$
x=u \mathrm{~T}^{2}
$$

we have used the values $\mathrm{T}^{2}=2,4,6,8$, and 10 (consequently, $x=1,2,3,4$, and 5 when $y=0$ and $u=a_{\max }=.5 \mathrm{~m} / \mathrm{sec}$ ). From Figs. 2 and 3 it is clear that, for a constant value of $T$, the final location of the platform sweeps through the first quadrant on a first arc as the parameter $\tau$ increases from zero and, for increasing values of $\mathrm{T}$, the first arcs continuously expand toward higher values of $(x, y)$. Thus, each point in the first quadrant can be reached by a three-switch-time trajectory ending on a first arc, and there is a one-to-one relationship between the points in the first quadrant and the parameter set $(T, \tau)$ which uniquely defines the trajectories reaching the first arcs.

Each first arc is uniquely defined by its value of $T$ and the range of the parameter $\tau$ required to sweep the first quadrant: $0 \leq \tau \leq \tau^{m}$, where the value $\tau^{m}(\mathrm{~T})$ corresponds to the intersection with the $y$ axis. The ratio of $\tau^{m}$ and $\mathrm{T}$ is displayed in Fig. 4 as a function of T. As discussed previously with Eqs. (40) and (50), there is a minimum half time $T_{\min }$ required to rotate 90 degrees. For the robot parameter values used in the figures, $T_{\min }=1.095$. When $T$ is less than $1.095, \tau^{\mathrm{m}}$ is not defined. When $\mathrm{T}$ is equal to $1.095, \tau^{m}=\mathrm{T}$ and the ratio is 1.0. As $\mathrm{T}$ increases, the ratio decreases as expected, reaching 0.35 when $\mathrm{T}=2.0$ and 0.09 when $\mathrm{T}=4.0$.

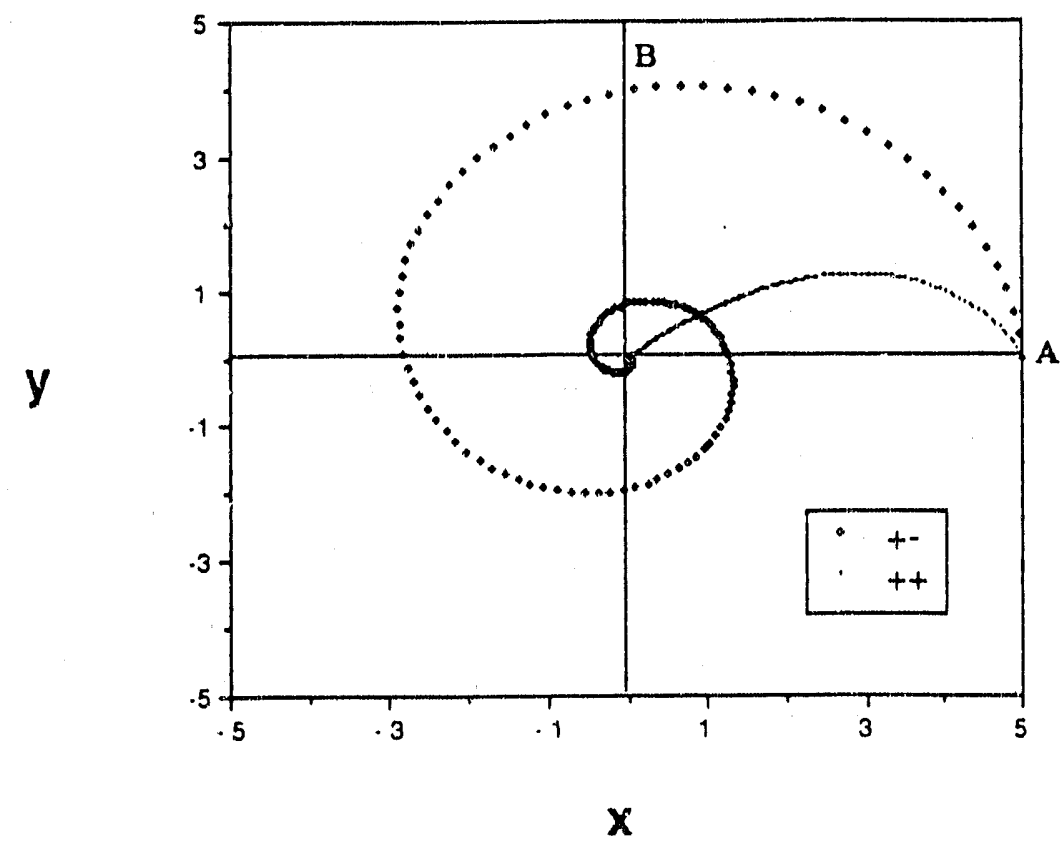

Fig. 2. Points that can be reached by trajectories with three switch times for a constent value of $T=\sqrt{10}$ sec. 


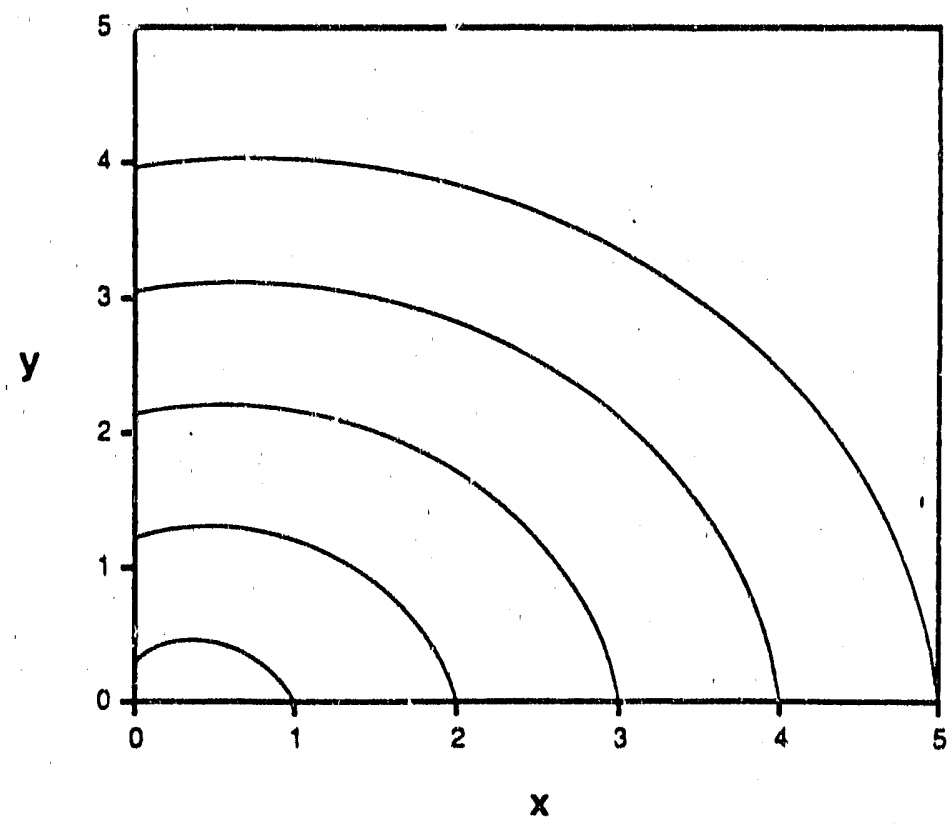

Fig. 3. Curves showing the location of the furthest points in the first quadrant that can be reached by trajectories with thres switch times for soveral values of $T\left(T^{2}=2,4,8,8\right.$, and 10$)$.

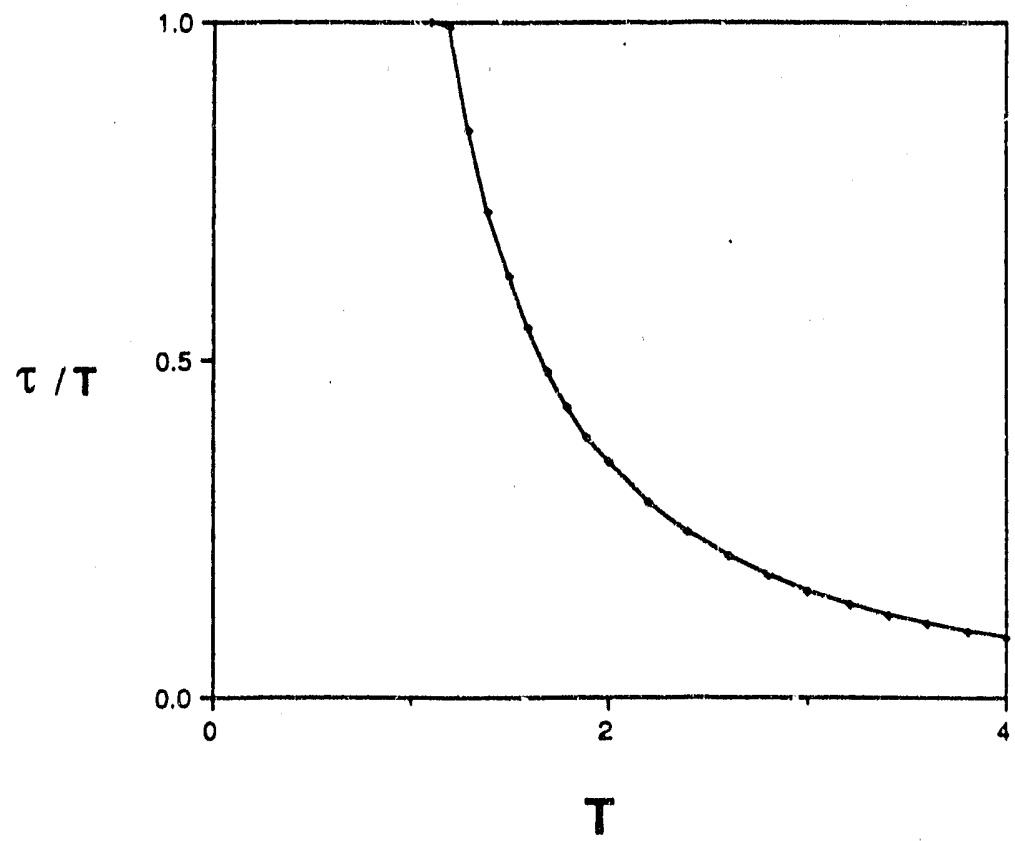

Fig. 4. Curve showing the ratio of $r^{m}$ and $T$. The sets $\left(T^{m}, T\right)$ define the intersection of the first arcs with the $y$ axis. 
Based on the above considerations, the remaining set of three-switch-time trajectories that are candidates for time-optimal trajectories to a point, is the uniquely defined set of trajectories reaching points on first arcs. Sample Cartesian paths of the robot for the cundidate three-switch-time trajectories are displayed in Fig. 5 for the case where $T^{2}=10$. Note that, at the end of all paths, both wheels have the same velocity and the Cartesian paths are line segments.

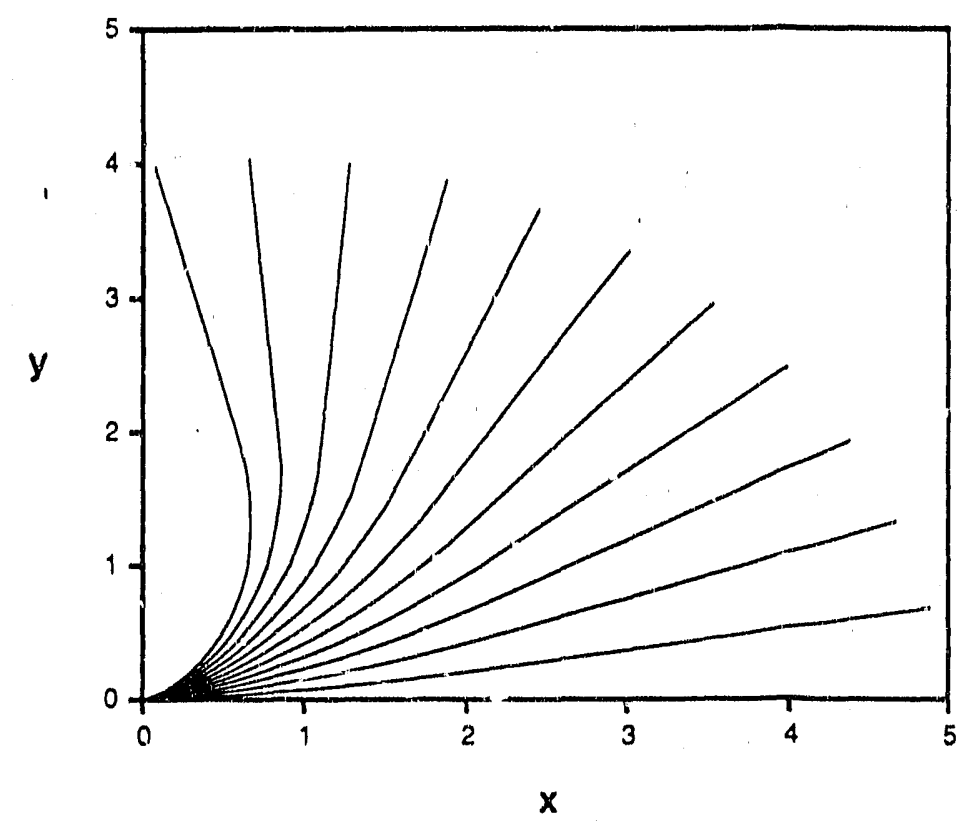

Fig. 5. Cartesian paths of the robot for three-switch-time trajectories ending on the first arc defined by $\mathrm{T}^{2}=10$.

\subsection{TRAJECTORIES TO A CONFIGURATION}

In a similar manner, and based on the discussion of Section 4.2 , we can use the parameterization developed in 4.1 to explore the space of four-switch-time trajectories when the goal is to reach a given configuration. In this case, the problem is not symmetrical about the $x$ and $y$ axes and the four quadrants have to be explored.

A four-switch-time trajectory can have either two swritch times for each wheel (Type 22) or one switch time for one wheel and three switch times for the other wheel (Type 13). For the Type 22 trajectories, the segment lengths satisfy: $\tau_{3}=$ $\tau_{4}=\mathrm{T}, \quad \tau_{7}=\tau_{8}=\tau_{9}=\tau_{10}=0, \tau_{1}+\tau_{3}=\mathrm{T}$ and $\tau_{2}+\tau_{6}=\mathrm{T}$. With these conditions and the constraint on the final orientation given by Eqs. (38), (42), and (45), these trajectories can be defined with two parameters, e.g., $\tau_{1}$ or $\tau_{2}$, and T. Similarly, for the Type 13 trajectories, the segment lengths satisfy: $\tau_{1}=\tau_{3}=\mathrm{T}, \tau_{5}=\tau_{7}=$ $\tau_{9}=\tau_{10}=0, \tau_{2}+\tau_{6}=\mathrm{T}$, and $\tau_{4}+\tau_{8}=\mathrm{T}$ (one switch time on the right wheel 
and three on the left) or: $\pi_{2}=\pi_{4}=\mathrm{T}, \quad \pi_{6}=\pi_{8}=\pi_{9}=\pi_{101}=0 ; \pi_{1}+\pi_{5}=\mathrm{T}$, and $\pi_{3}+\pi_{7}=\mathrm{T}$ (three switch times on the right wheel and or on the left). With the constraint on the finall onientation, these trajectories can iso be defined with two parameters $\pi_{2}$ or $\pi_{4}$, and $T$; or $\pi_{11}$ or $\pi_{3}$, and $T$.

Figure 6 displays the points: that can be reached by four-switch-time trajectories when the orientation is $\phi=0,8$ radians and $T^{2}=10$. Since we thave two types of trajectories and four combinations of initiall acceleration $(++,--,+-,-+)$, there are eight curve segmentis displayed in Fig. 6. There are four significant intersection. poinds $(C, D, E, F)$ in the figure: These are points where the four-swritch-time trajectionies become three-switch-time trajectories of the Type 12 , i.e., where one of their segment $\pi_{i}$ vanishers. For example, the two points, $C$ and $D$, in the firsti quadrant are where the two curves in Fig. 2 reach an orienthation of 0.8 radians (at, the points, Eqs. (491)) and (50)) are satisfied). All of the eight curve stginents start at one of these four points and end at another. The same pattiern is observed in Figs. 7 and 8: which display the points that can be reached by four-switch-time trajectiories when the final orientation is $\phi=1.57$ radians and $\phi=3.14$ radians $\left(\right.$ and $\left.T^{2}=10\right)$.

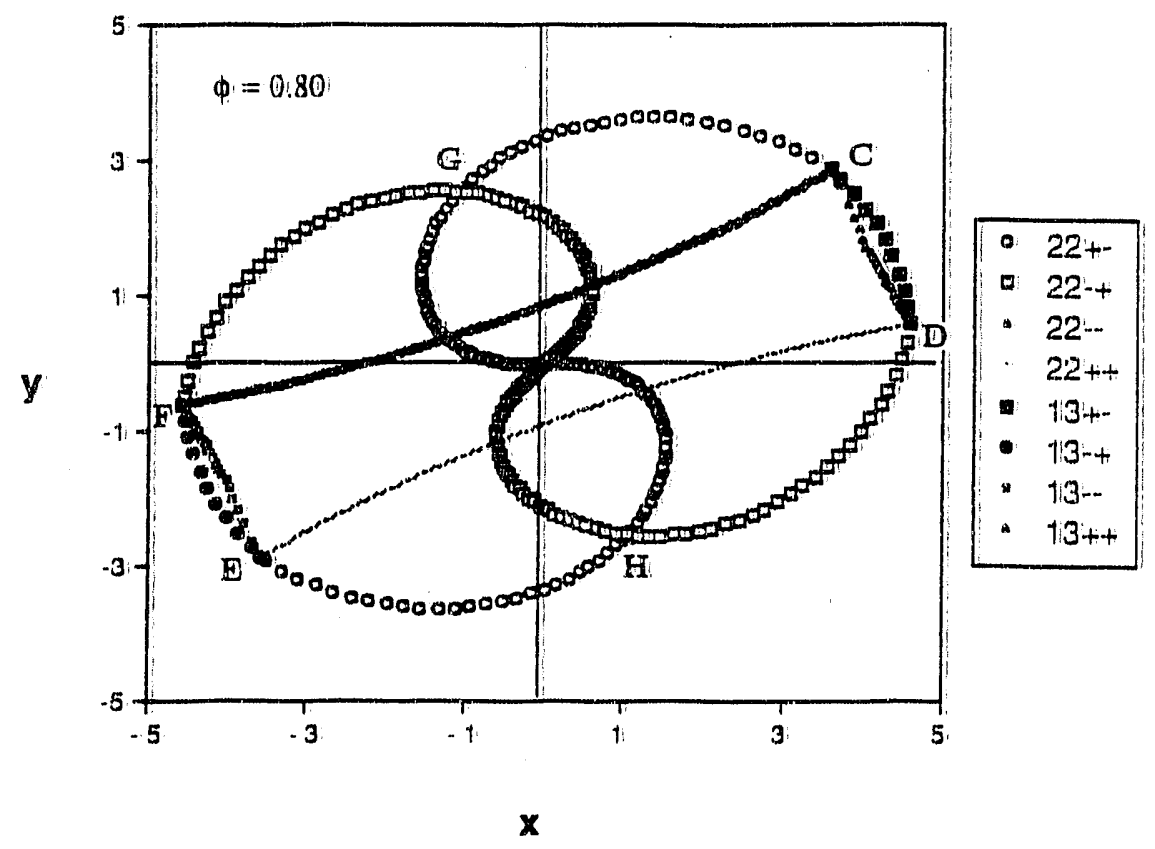

Fig. 6. Points that can be reached by trajectories with four switch times for a constant value of $T\left(T^{2}=10\right)$ when the final orientation is 0.8 radians. 


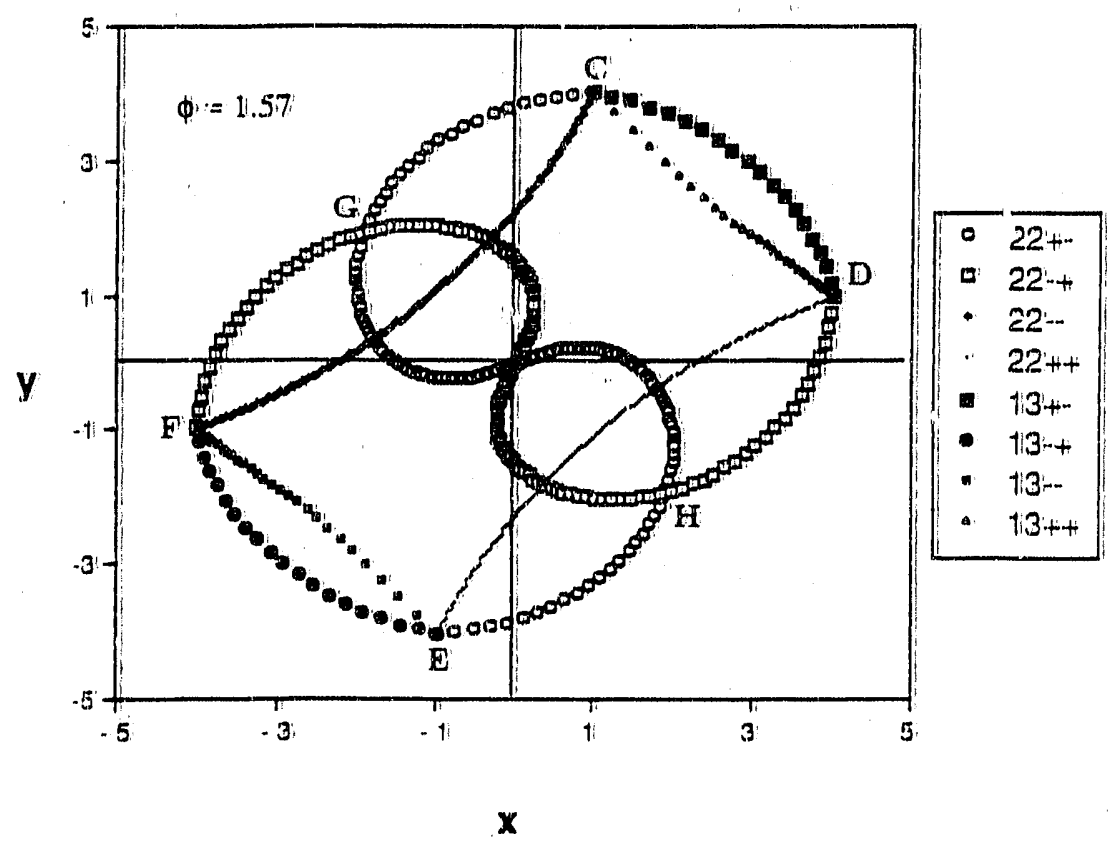

Fig. 7. Points that can be reached by trajectories with four switch times for a constant value of $T\left(T^{2}=10\right)$ when the final oriextation is 1.57 radians.

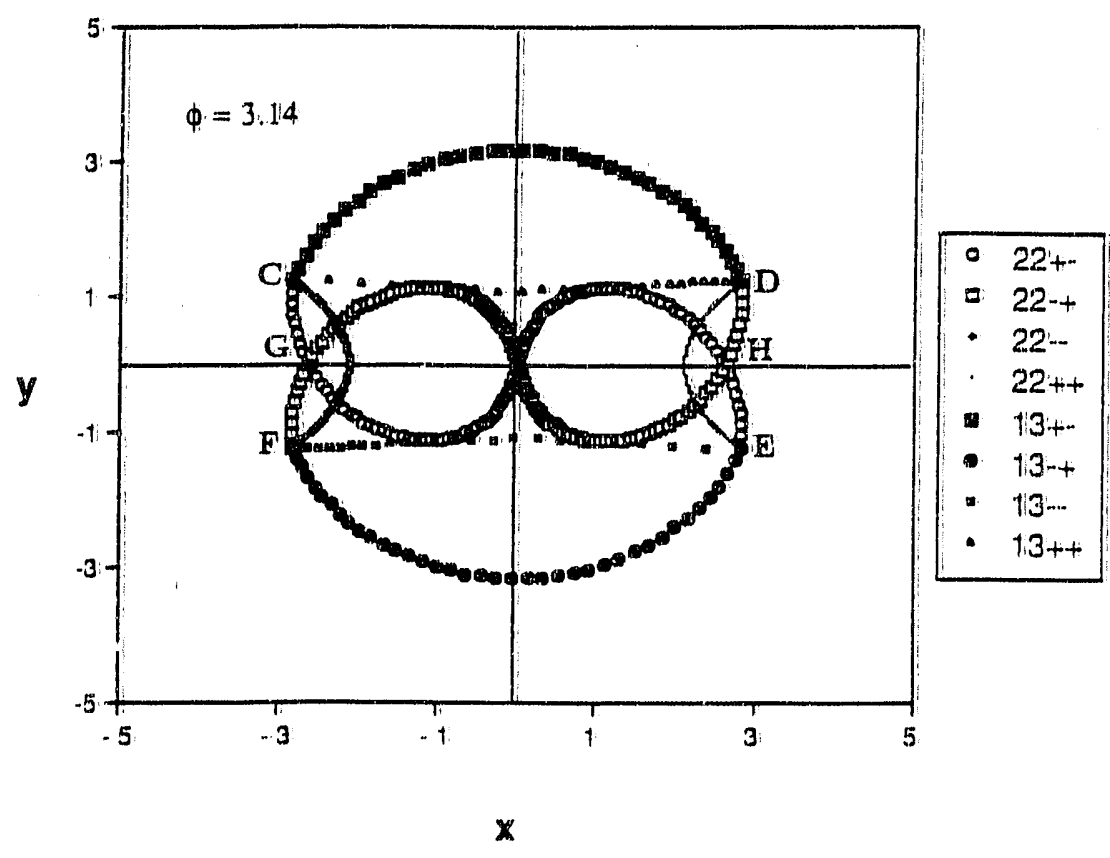

Fig. 8. Points that can be reached by trajectories with four switch times for a constant value of $T\left(T^{2}=10\right)$ when the final orientation is 3.14 radians. 
Figures 6 and 7 are not symmetrical about the $x$ or $y$ axis. However, we can show that the two axes of symmetry are rotated by $\phi / 2$ (thus, Fig. 8 is symmetrical about the $x$ and $y$ axis); and identify the location of the points $G$ and $H$. Consider an. arbitrary bang-bang trajectory that starts att $(0,0,0)$ and reaches $\left(x^{1}, y^{1}, \phi\right)$. Suppose that a second trajectory leaves $(0,0,0)$ with the time reverse control trajectory (the length of the initial segment of the second trajectory will be equal to the length of the final segment of the first trajectory but the signs of the control variables will be reversed). The holonomic variables (wheel rotation and finall orientation): will De the same for the two trajectories but the nonholonomic variables $(x, y)$ will be different. Thus, the second trajectory will reach $\left(x^{2}, y^{2}, \phi\right)$.

If we let time run backwards, the second trajectory is identical to the first and we can derive a mapping from $\left(x^{1}, y^{1}\right)$ to $\left(x^{2}, y^{2}\right)$ :

$$
\begin{aligned}
& x^{2}=x^{4} \cos \phi+y^{1} \sin \phi \\
& y^{2}=x^{1} \sin \phi-y^{1} \cos \phi
\end{aligned}
$$

The mapping applies to any arbitrary bang-bang trajectory. On thie first line of syminetry, the second point is the same as the first $\left[\left(x^{1}, y^{1}\right)=\left(x^{2}, y^{2}\right)=(x, y)\right]$ :

$$
y / x=\sin \phi /(1+\cos \phi)=(1-\cos \phi) / \sin \phi=\tan (\phi / 2)
$$

Thus, the direction of the first line of symmetry is $\phi / 2$. On the second line of symmetry, the second point is. reflected through the origin $\left[\left(x^{1}, y^{1}\right)=\left(-x^{2},-y^{2}\right)=\right.$ $(x, y)]$ :

$$
y / x=-(1+\cos \phi) / \sin \phi=-\sin \phi /(1-\cos \phi)=\tan [(\pi+\phi) / 2]
$$

Thus, the direction of the second line of symmetry is $(\pi+\phi) / 2$.

We can move the final configuration across the first line of symmetry by time reversing the control trajectory. We can move the final configuration across the second line of symmetry by reversing the control trajectory between the wheels and by changing the signs of the controls (if we do not reverse the signs of the wheel rotations, the sign of the final orientation will be reversed).

Just as in Section 5.1, we can define a set of candidates for time-optimality as the set of trajectories reaching furthest in the plane for a given value of $T$. $F \quad n$ Figs. 6 to 8, it can be seen that the envelop ( $C D H E F G C)$ is reachec by trajectories of Type $13+-, 13-+$, and some of the Type $22+-$ and $22-+$ that are uniquely defined by the points $G$ and $H$. 


\section{BANG.BANG TRAJECTORIES}

Just like in Section 5.1, we can also show that the candidate four-switch-time trajectories can reach any configuration by varying the half time parameter $T$. Figures 9 and 10 illustrate the continuous sweeping through all of the points in the plane as: the parameter $T$ increases. In Fig. 9 the final orientation is $\phi=$ 0.80 radiarus;, while in Fig. 10 the final orientation is $\phi=3.14$ radians. In both figures, the curves are for $\mathrm{T}^{2}=2,4,6,8$, and 10 , and $a_{\max }=0.5$ meters $/$ second. $^{2}$ Figure 10 includes only four sets of curves (rather than five like Fig. 9) because the time required for a pure rotation of 3.14 radiams $\left(\mathrm{T}^{2}=2.4\right.$ ) is longer than the first value for the parameter $\mathrm{T}\left(\mathrm{T}^{2}=2\right)$.

Sample Cartesian paths of the robot for some of these candidate time-optimal four-switch-time trajectories to a configuration are displayed (for the case where $\mathrm{T}^{2}=10$ ) in Figs. 11 and 12 for final orientations of $\phi=0.8$ radians and $\phi=3.14$ radians, respectively. Note that, as the control trajectories evolve from Type $22-+$ to $13+-$ to $22+-$, the Cartesian trajectories change continuously.

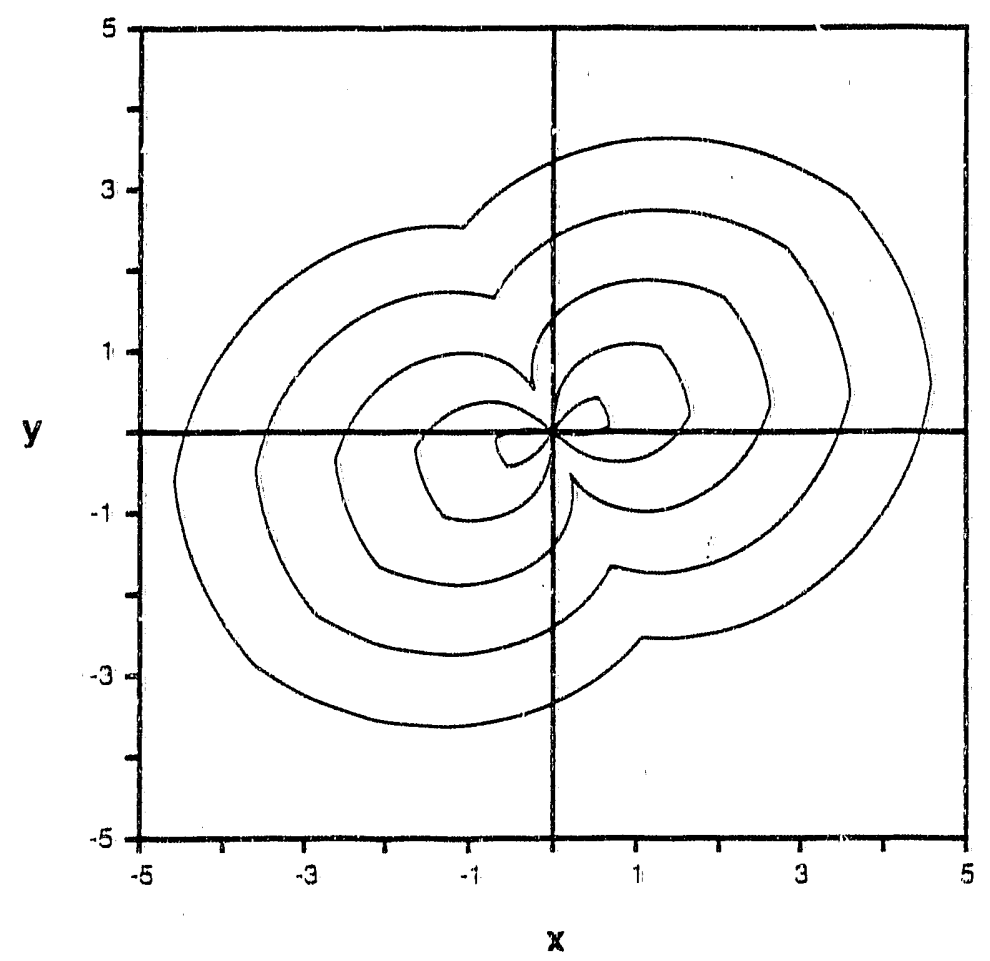

Fig. 9. Curves showing the location of the furthest points that can be reached by trajectories with four $s$ writch times for several values of $T\left(T^{2}=2,4,6,8\right.$, and 10) when the final orientation is 0.8 radians. 


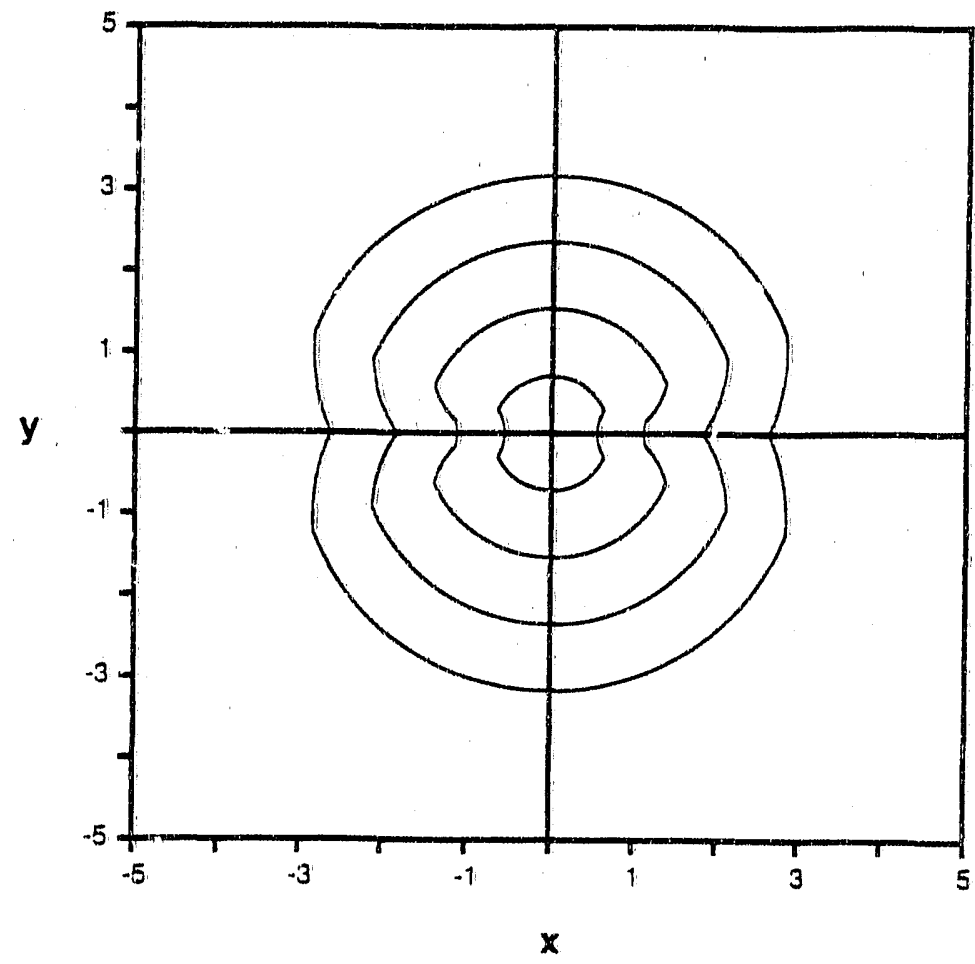

Fig. 10. Curves showing the location of points that can be reached by trajectories with four switch times for several values of $T\left(T^{2}=4,6,8\right.$, and 10) when the final orientation is 3.14 radians.

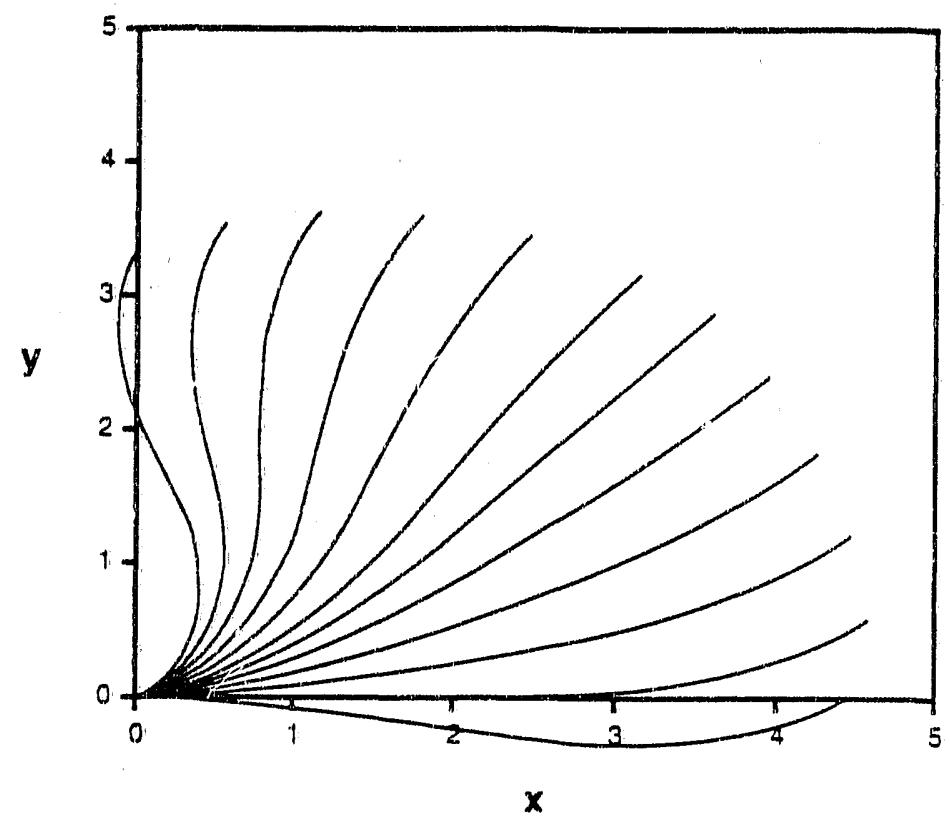

Fig. 11. Cartesian trajectories for paths with four switch times for a constant value of $\mathrm{T}\left(\mathrm{T}^{3}=10\right)$ when the final orientation is 0.8 radians. 


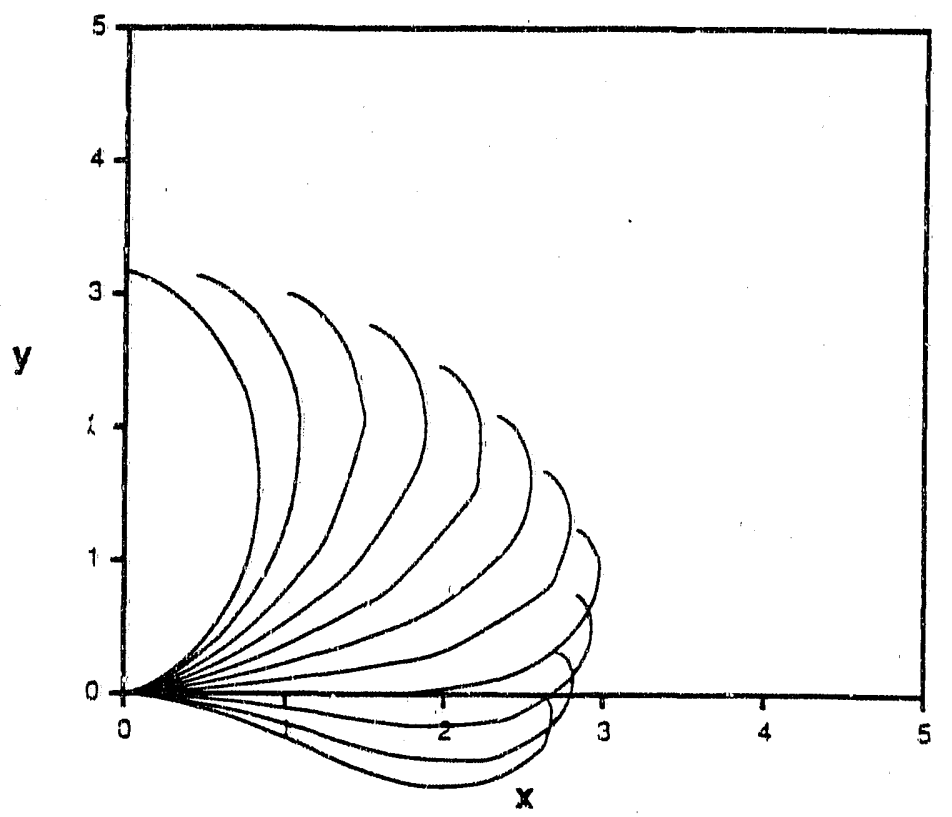

Fig. 12. Cartesian trajectories for paths with four switch times for a constant value of $T\left(T^{3}=10\right)$ when the final orientation is 3.14 radians. 


\section{OPTIMAL TRAJECTORIES}

In this section, we show that the candidate three-switch-time trajectories identified in the previous section provide time optimal paths to a position, and the candidate four-switch-time trajectories provide time optimal paths to a configuration.

The maximum principle provides necessary (but not sufficient) conditions for an optimal trajectory. To show that a bang-bang trajectory is optimal, we must show that it satisfies the necessary conditions and that it is better than altecnative paths. Indeed, we will find that some trajectories satisfy the necessary conditions but are not optimal. Since the candidate sets of trajectories have been identified through comparisons with trajectories involving the minimum needed number of switch times, we show that they are better than alternative paths by comparing them to trajectories with greater numbers of switch times.

To verify that a bang-bang trajectory satisfies the necessary conditions, we must calculate the dual variables and show that they are consistent with the control paths [that $u_{1}$ is positive (negative) when $\psi_{4}$ is positive (negative), etc.]. In Section 4, we defined four auxiliary variables $\left(z_{i}\right)$ that can be used to calculate the initial conditions for the dual variables. Following the procedure described in section four, we can calculate the dual variables for any bang-bang solution.

We will consider first the three switch time trajectories to a position. Consider the results shown in Fig. 2. Our subset of candidate time-optimal-trajectories includes the trajectories of the Type $12+-$ that end on the first arc, i.e., the portion of the curve that lies in the first quadrant. We have calculated the dual variables for a wide range of trajectories and found that all $12+-$ trajectories that end on first arcs satisfy the necessary conditions. Typical results for a trajectory defined by $(T, \tau)=(\sqrt{10}, .4)$ are displayed in Fig. 13. The trajectory reaches the point $(0.66,4.03)$. For the right wheel, the acceleration is positive for $(0.0$ to 3.16$)$ and negative for $(3.16$ to 6.32$)$. The corresponding dual variable $\left(\psi_{4}\right)$ is found positive for $(0.0$ to 3.16$)$ and negative for $(3.16$ to 6.32$)$. For the left wheel, the acceleration is negative for $(0.0$ to 0.40$)$, positive for $(0.40$ to 3.56$)$ and negative for (3.56 to $6.32)$. The corresponding dual variable $\left(\psi_{5}\right)$ is found negative for ( 0.0 to 0.40 ), positive for ( 0.40 to 3.56 ) and negative for ( 3.56 to 6.32 ). Finally, the transversality condition requiring that $\psi_{3}=0.0$ at the end of the trajectory is seen to be verified.

We have also calculated the dual variables for the other $12+-$ trajectories, and it is interesting to note that they, too, satisfy the necessary conditions except for those that reach points on the spiral after it reenters the first quadrant (and the $12+-$ trajectories cross the $12++$ trajectories). On the other hand, none of the $12++$ trajectories satisfy the necessary conditions. 


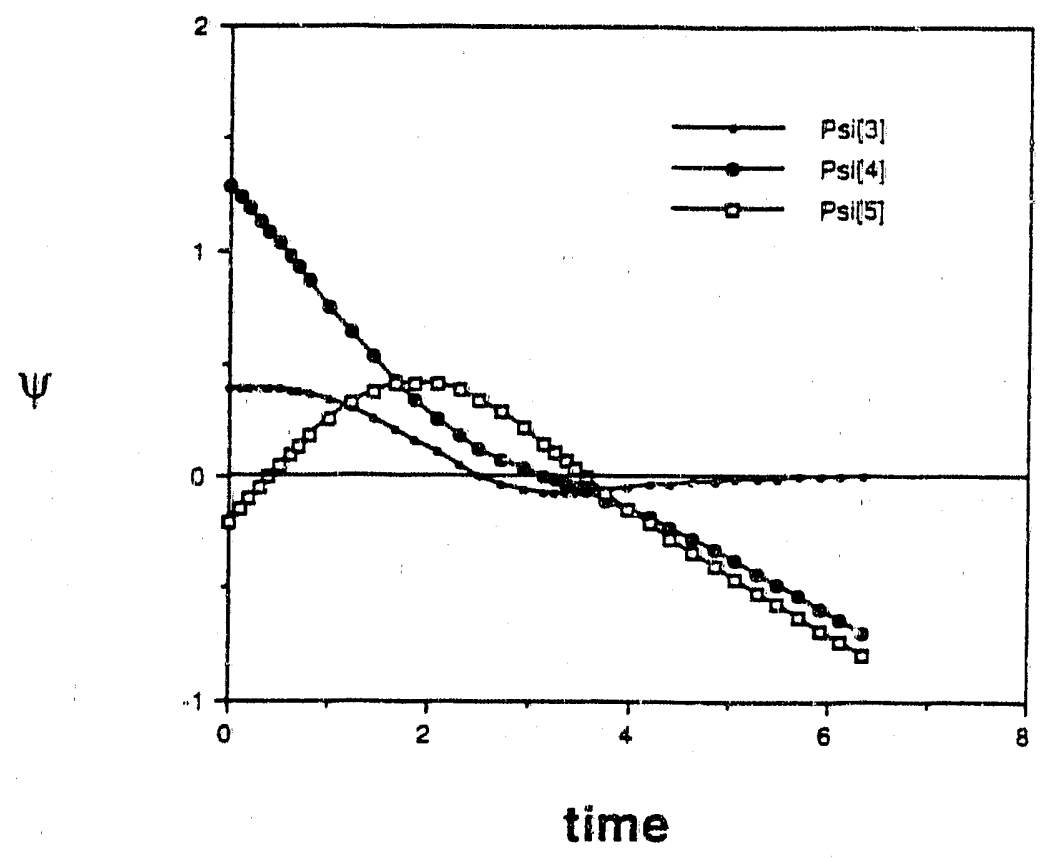

Fig. 13. Dual variables for a three-switch-time trajectory leading to the point $(0.66,4.03)$.

In a similar fashion, we have calculated the dual variables for a wide range of candidate time-optimal trajectories to a configuration, i.e., those four-switch-time trajectories that end at a configiration laying on $C D H E F G$ envelops of the type shown in Fig. 6, and have found that they satisfy the PMP necessary conditions. Typical results are displayed in Fig. 14 for a $22+-$ trajectory corresponding to $\phi=0.8$ radians, $T^{2}=10$ and $\tau_{1}=2.84$. The trajectory reaches the configuration $(\tilde{x}, y, \phi)=(0.20,3.43,0.8)$. For the right wheel, the acceleration is positive for $(0.0$ to 2.84$)$, negative for $(2.84$ to 6.00$)$, and positive for (6.00 to 6.32). The corresponding dual variable $\left(\psi_{4}\right)$ is found respectively positive, negative, and positive on the same interval. For the left wheel, the acceleration is negative for $(0.0$ to 0.52$)$, positive for $(0.52$ to 3.68$)$ and negative for ( 3.68 to 6.32$)$, and the corresponding dual variable $\left(\psi_{5}\right)$ is found appropriately negative, positive, and negative on the same intervals.

In the remainder of this section, we numerically demonstrate that the trajectories of the candidate sets are time-optimal by showing that they are faster than trajectories with more switch times. If the total trajectory time $(T)$ is fixed, an optimal trajectory will reach further from the origin than any other trajectory. We demonstrate numerically that the "best" trajectories with $n$ switch times are longer than the "best" trajectories with $n+1$ switch times. 


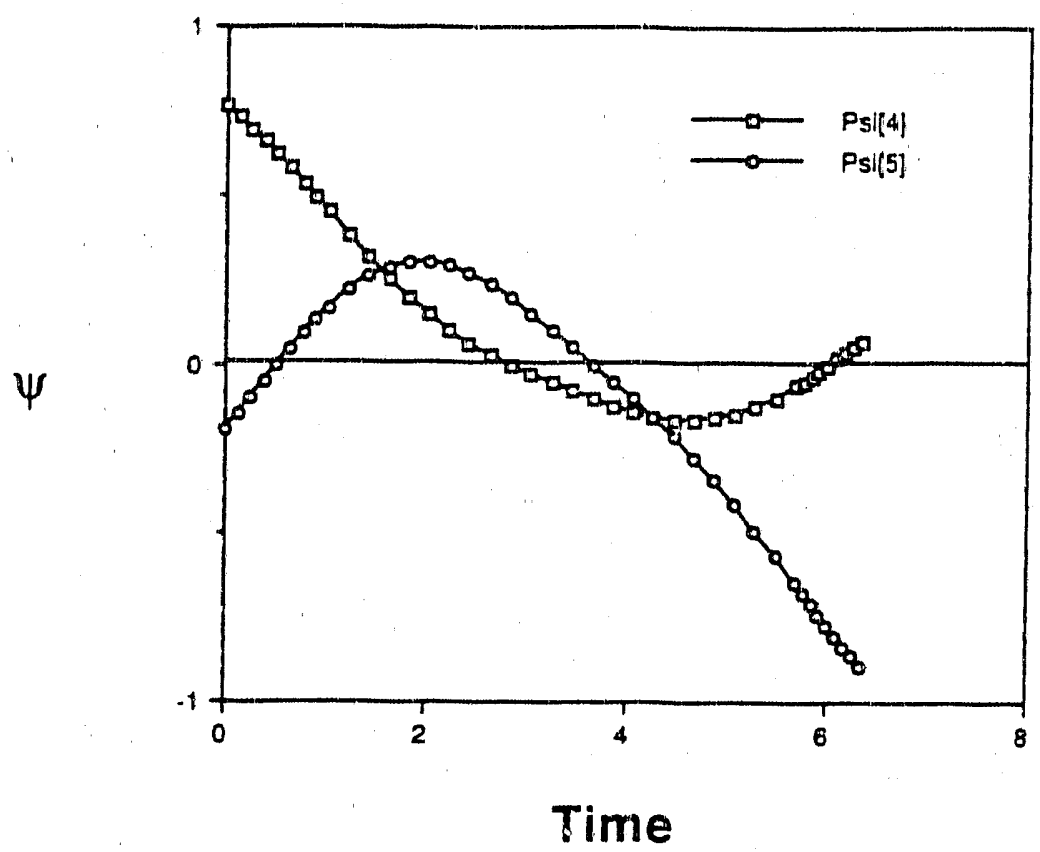

Fig. 14. Dual variables for a four-switch-time trajectory leading to the configuration $(0.20,3.43,0.80)$.

A trajectory with a total of $n$ switch times $\left(n_{R} \geq 1\right.$ on the right wheel, $n_{L} \geq 1$ on the left wheel, $n_{R}+n_{L}=n$ ) involves $n+2$ time segments, $\tau_{i}$, which are distributed on the two wheels with at least two segments on each wheel. Any $n$-switch-time trajectory (with $n+2$ segments) of type $r_{R} n_{L}$ can be considered as an $n+1$-switcintime trajectory (with $n+3$ segments) of type $\left(n_{R}+1\right) n_{L}$ or type $n_{R}\left(n_{L}+1\right)$ in which the additional time segment is set to zero. If we denote this additional time segment by $\rho$ and use it as an additional trajectory parameter that can vary over its entire feasible range within $[0, T]$, then all $n+1$-switch-time trajectories can be evolved from their parent $n$-switch-time trajectory. These can then be compared to ascertain that the parent trajectories always reach further than their "offspring" for a same total trajectory time.

First conjider the special case when $n=2$. The "best" trajectories with two switch times are pure translation (as opposed to the pure rotation trajectories which do not leave the origin). These trajectories are part of the candidate sets, although they only reach points on the $x$ axis. However, it is clear from Fig. 2 that when the parameter is varied, the two-switch-time trajectories reach further than any threeswitch-time trajectories (or trajectories with any greater number of switch times) that end on the $x$ axis. Thus, to reach points on the $x$ axis, these two-switch-time trajectories clearly are the time-optimal ones. 
Consider the case when $n=3$. The three switch trajectories in Fig. 2 have one switch time on the right wheel and two switch times for the left wheel, i.e., they are of Type 12. They have two types of offspring trajectories which are the only two types of four-switch-time trajectories: Type 22 and Type 13. In this case, the parameter $\rho$ becomes either the third time segment on the right wheel or the fourth time segment on the left wheel. When the parameter is zero, the four-switch-time trajectories reduce to the three-switch-time trajectories. As the parameter increases, the offspring trajectories spanned the space of four-switch-time trajectories.

Offspring four-switch-time trajectories are compared to the parent three-switchtime trajectories in Fig. 15, for Type 22, and Fig. 16 for Type 13. In both cases, results for the parameter increasing from 0.0 to 0.3 are displayed. As the parameter increases, the end points of the four-switch-time trajectories move monotonicaily toward the origin. Thus, the best three-switch-time trajectories always reach further from the origin than the best four-switch-time trajectories and the distance between the end points increases as the four switch paths become less like the three switch paths.

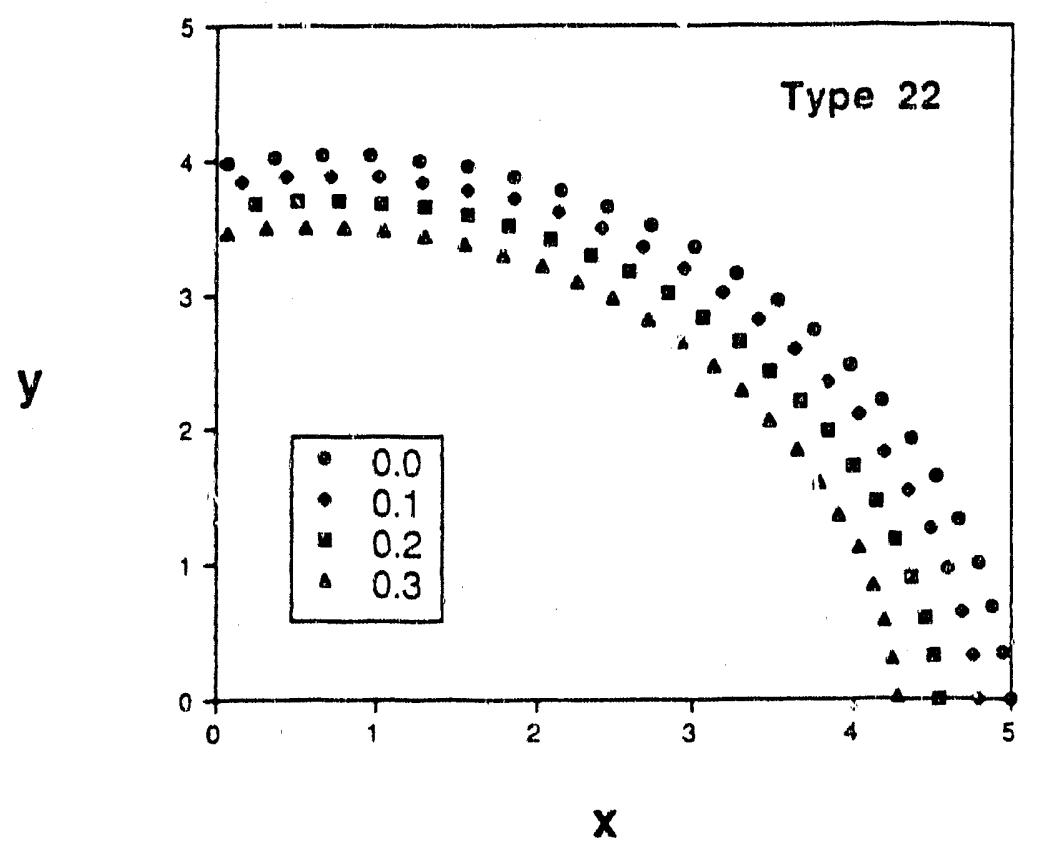

Fig. 15. Comparison of four-switch time trajectories of Type 22 and three-switchtime trajectories. 


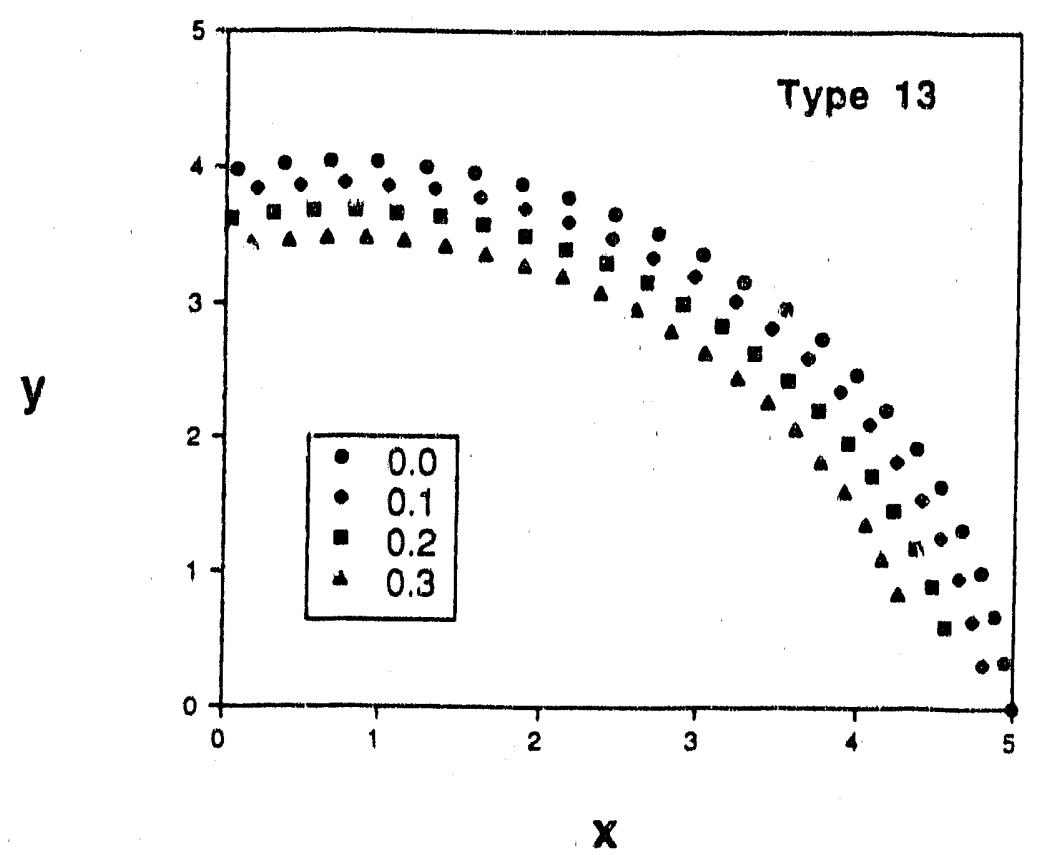

Fig. 16. Comparison of four-switch-time trajectories of Type 13 and three-switchtime trajectories.

Consider the case when $n=4$. There are four ijpes of five-switch-time trajectories: Type 14, Type 23, Type 32, and Type 41, which can all be found from the parents four-switch-time trajectories of Type 13 and 22, and compared to them to show that they are not time optimal. Examples of the comparionn results are illustrated in Figs. 17 to 21. The Type 23+- trajectories are compared to the 'Type 22+- trajectories in Fig. 17. As the parameter increases from 0.0 to 0.3, the end points of the Type 23+- trajectories in the upper half plane move monotonically toward the origin. They also move toward the $x$ axis in the first quadrant, but they always remain inside the Type $13+-$ and Type $22++$ curves.

The Type 23+- trajectories are compared to the Type 13+- trajectories in Fig. 18. As the paranneter increases from 0.0 to 0.3 , the Type $23+-$ trajectories move monotonically toward the origin. They also move radially but they always remain inside the Type $22+-$ and Type $22++$ curves.

The Type $23++$ trajectories are compared to the Type $13++$ trajectories in Fig. 19. As the parameter increases from 0.0 to 0.3 , the end points of the Type $23++$ trajectories move monotonically toward the origin. They also move radially but they always remain inside the Type $22+-$ and Type $22++$ curves. 


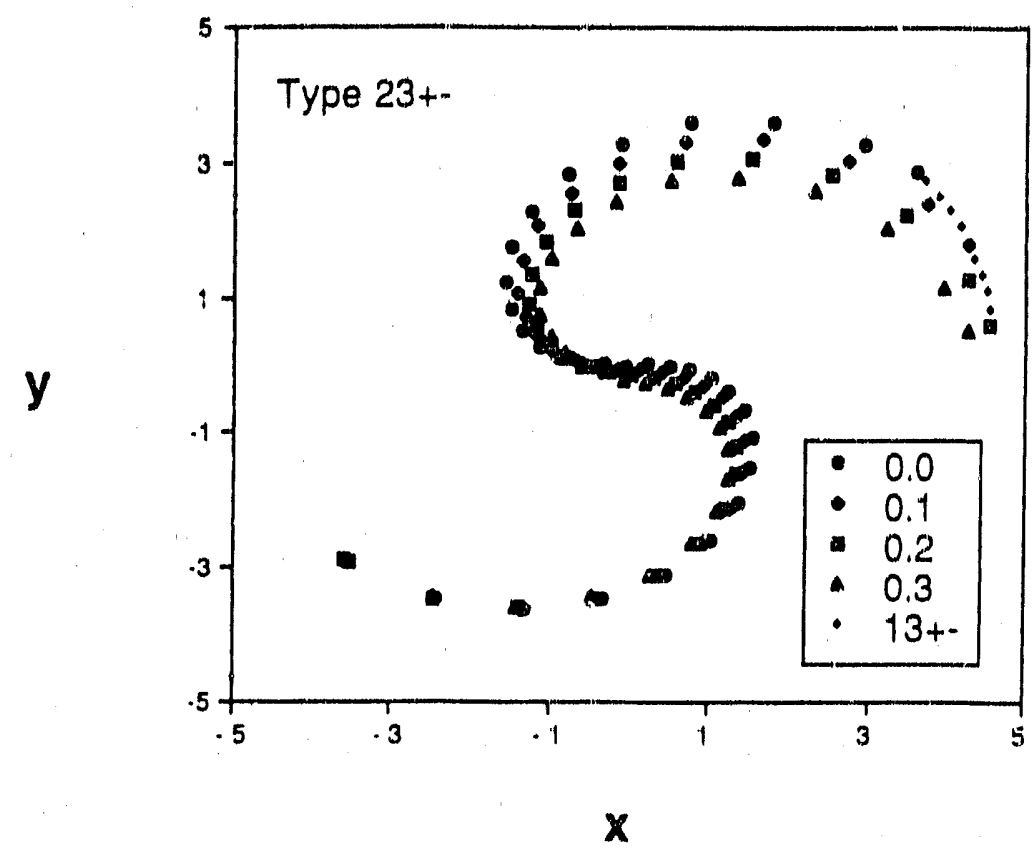

Fig. 17. Compurison of five-switch-tine trajectories of Type 23+- and fourswitch-time trajectories of Type 22+--.

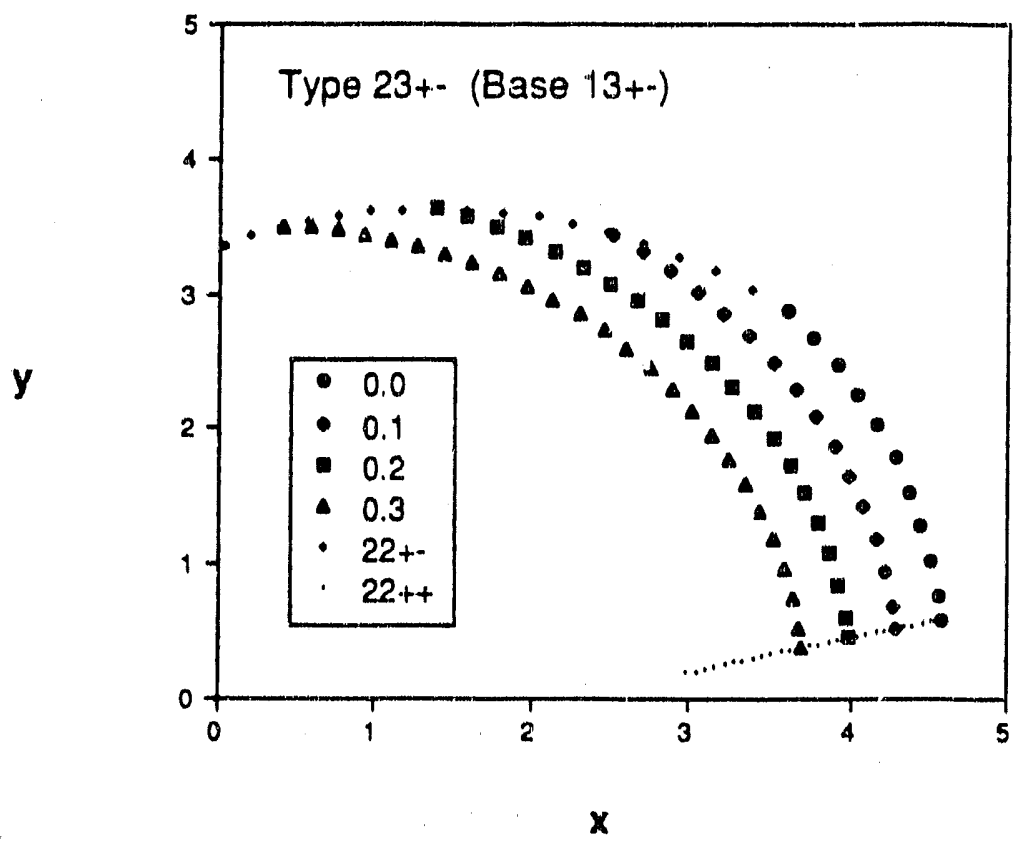

Fig. 18. Comparison of five-switch-time trajectories of Type $23-t-$ and fourswitch-time trajectories of Type 13+-. 


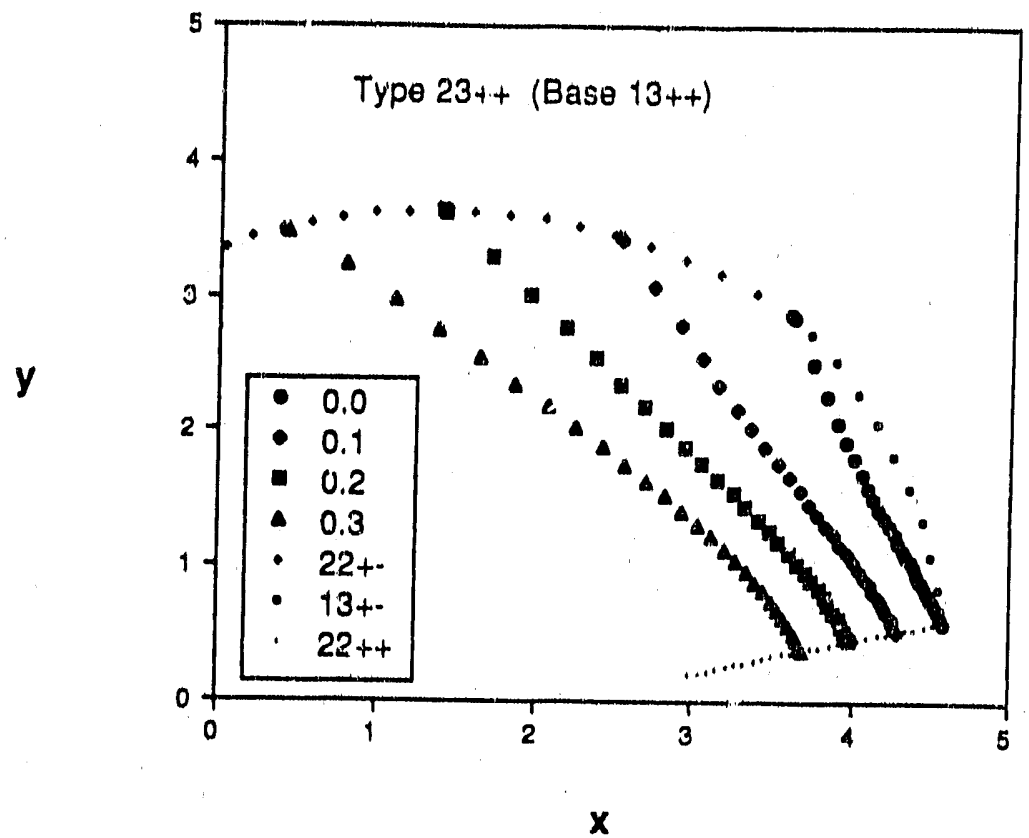

Fig. 19. Comparison of five-switch-time trajectories of Type $23++$ and fourswitch-time trajectories of Type $13++$.

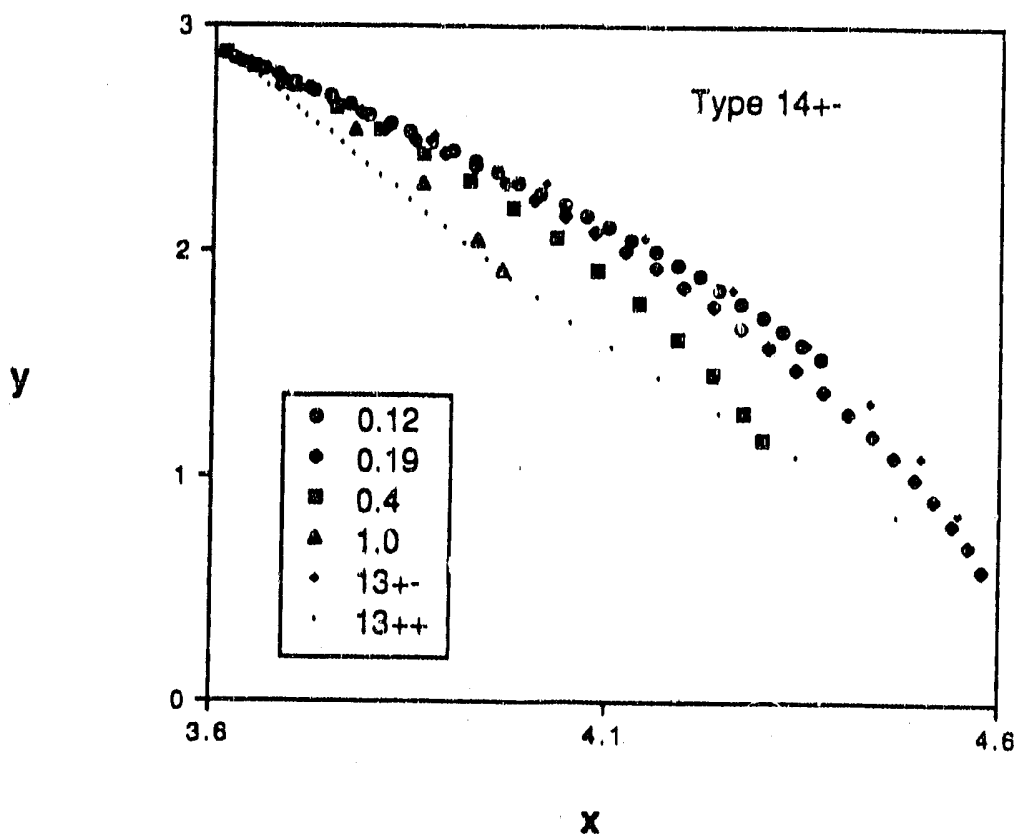

Fig. 20. Comparison of five-switch-time trajectories of Type 14t- and fourswitch-time trajectories of Type 13. 


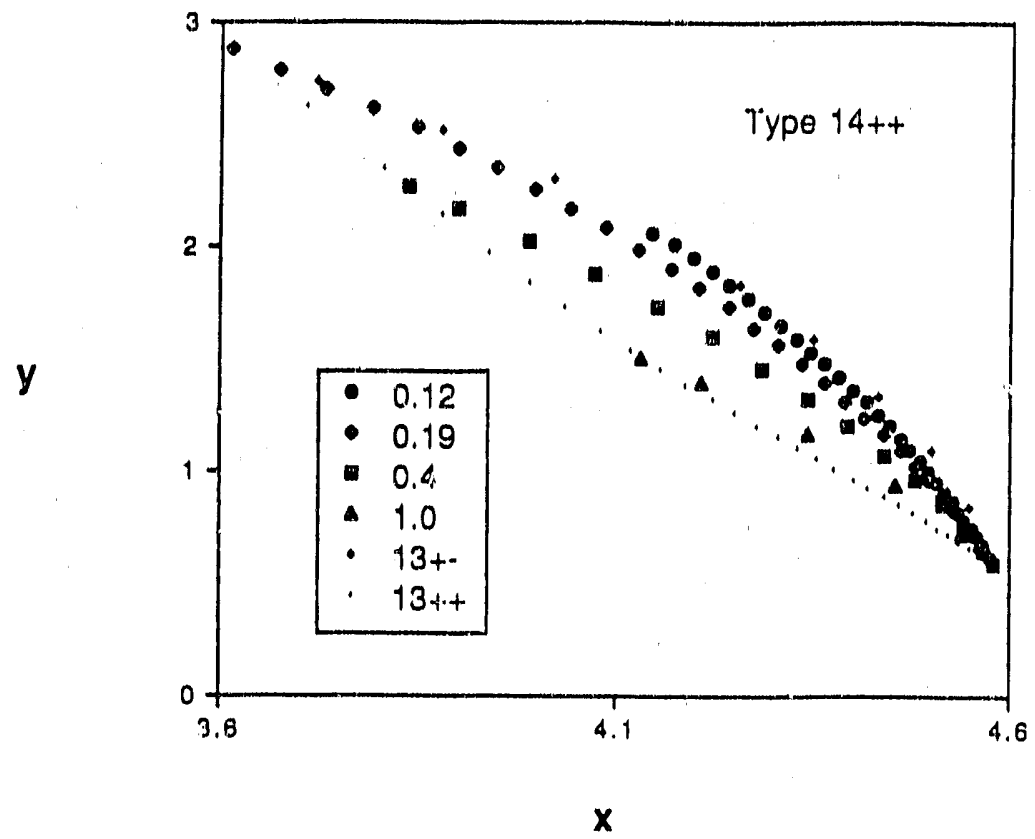

Fig. 21. Comparison of five-switch-time trajectories of Type 14t+ and fourswitch-time trajectories of Type 13.

The Type 14+- and Type $14++$ trajectories are compared to the Type $13+-$ and Type 13++ trajectories in Figs. 20 and 21. The end points of the Type 14 trajectories occupy the inside of the lens shaped region defined by the Type 13 trajectories. As the parameter increases from 0 to 1.0, the end points of the Type 14 trajectories sweep the interior of the lens with a upper left fixed point for the case of Fig. 20, and a lower right fixed point for the case of Fig. 21.

Similar families of curve can be easily generated to compare parents and offspring trajectories with five switch times and greater. The important general result of such an exercise is that the best offspring trajectories never reach further than their parent best candidate for a same allowed total trajectory time.

In summary, any point in the plane can be reached by a three-switch-time trajectory which has been uniquely defined in Section 5.1 and shown to be timeoptimal by reaching the desired $(x, y)$ position faster than other three-switch-time trajectories or trajectories with a higher number of switch times. Similarly, any configuration in the plane can be reached by a four-switch-time trajectory which has been uniquely defined in Section 5.2 and shown to be time-optimal by reaching the configuration faster than other four-switch-time trajectories or trajectories with a greater number of switch times. 


\section{USE OF THE METHOD FOR ROBOT TRAJECTORY PLANNING}

Given the resultis of the previous sections, the implementiation on our robots has focussed on developing the driver module for the robots, i.e., the module which detiermines, the contirol trajectories necessary for a time optimal motion of the platform between given subgoals in a sequence provided by the routie flanning module [e.g., see Vasseur, Pin and Tayylor (1992); or Andlersen et al. (1992)]. Each sultgoal is specified as a given configuration where the platform must stop for the robot to perform some sensing, manipulation, or other fasks. In the first st. ", of the algoritsm, the arrival configuration is expressed as a relative position and orientation with respect to the starting configuration. If a final orientation is not specified, then the time-optimal three-switch-time trajectory to a point is sought which, as explained in Section 5.1 , is characterized by two parameters. If a desired final orientation is specified, the time-optimal trajectory is a four-switchtime trajectiory to a configuration and, as discussed in Section 5.2, Eq. (40) is first used to reduce the number of unknown paramevers from three to two. In both cases, the same numerical search technique [Powell's method (Press (1988))], can thus be used to determine the two parameters $(T, \tau)$ for the given final location $((x, y)$ making use of the symmetries with respect to the $x$ and $y$ axes or $\phi / 2$ and $(\pi+\phi / 2)$ axes as appropriate. From $(\mathrm{T}, \tau)$ the switch times are easily calculated and the controls directly sent to the robot actuator servos.

As an example of the gain obtained with the time-optimal trajectories compared with minimum-length paths to a configuration (i.e., rotate, translate, rotate), Table 1 shows the total trajectory times required to reach the point $(3,3)$ at several orientations. $T_{\text {apt }}$ represents the optimal trajectory time, while $T_{r t r}$ is the time required to execute the RTR (rotate, trasslate, rotate) strategy with bang-bang controls. As the orientation increases from 0.80 to 3.14 radians, $\mathrm{T}_{\text {opt }}$ increases from 6.18 seconds to 7.15 seconds and the ratio, $R=\mathrm{T}_{\text {opt }} / \mathrm{T}_{\text {rtr, }}$, varies from 81 to .71. These results are typical of the gains obtained with optirnal paths over RTR paths, and average about $25 \%$ for trajectories to near configurations (less than 5 meters away from the start point). The gain, of course, decreases as the distance separating the two configurations increasec, as well as for trajectories approaching pure translations on the $x$ axis (for which $R=1$ ).

One of the concerns which usually arises when implementing time-optimal controls on a robot relates to the capability of the controller to closely approximate the bang-bang demands on the controls. For our 2,000 lbs. HERMIES-III robot, the results of well-tuned conventional PID wheel controllers (see Reister, 1992) proved extremely satisfaciory, as illustrated in Fig. 22. In the figure, the velocity target on one of the wheels and the actual velocity profile obtained during experimentis with the robois are displayed by the plain and dotted lines, respectively. The velocity target is a three-switch-tirne trajectory of the wheel with a value of $a_{\max }=.2 \mathrm{~m} / \mathrm{sec}^{2}$. With this very good behavior of the controller, time-optimal motions of the robot were realized with much less than $1 \%$ error. 
Table 1. Values of the optimal time $\left(\mathrm{T}_{\text {opt }}\right)$, the time for rotate-translaterotate $\left(\mathrm{T}_{r t_{r}}\right)$ with bang-bang controls, and their ratio $(R)$ for paths that reach the configuration $(3, \mathbf{3}, \phi)$.

\begin{tabular}{|c|c|c|c|}
\hline $\begin{array}{c}\phi \\
\text { Radians }\end{array}$ & $\begin{array}{c}\mathrm{T}_{\text {opt }} \\
\text { Seconds }\end{array}$ & $\begin{array}{c}\mathrm{T}_{\text {rtr }} \\
\text { Seconds }\end{array}$ & $\mathbf{R}$ \\
\hline 0.80 & 6.18 & 7.59 & .81 \\
1.57 & 6.36 & 8.92 & .71 \\
3.14 & 7.15 & 10.06 & .71 \\
\hline
\end{tabular}

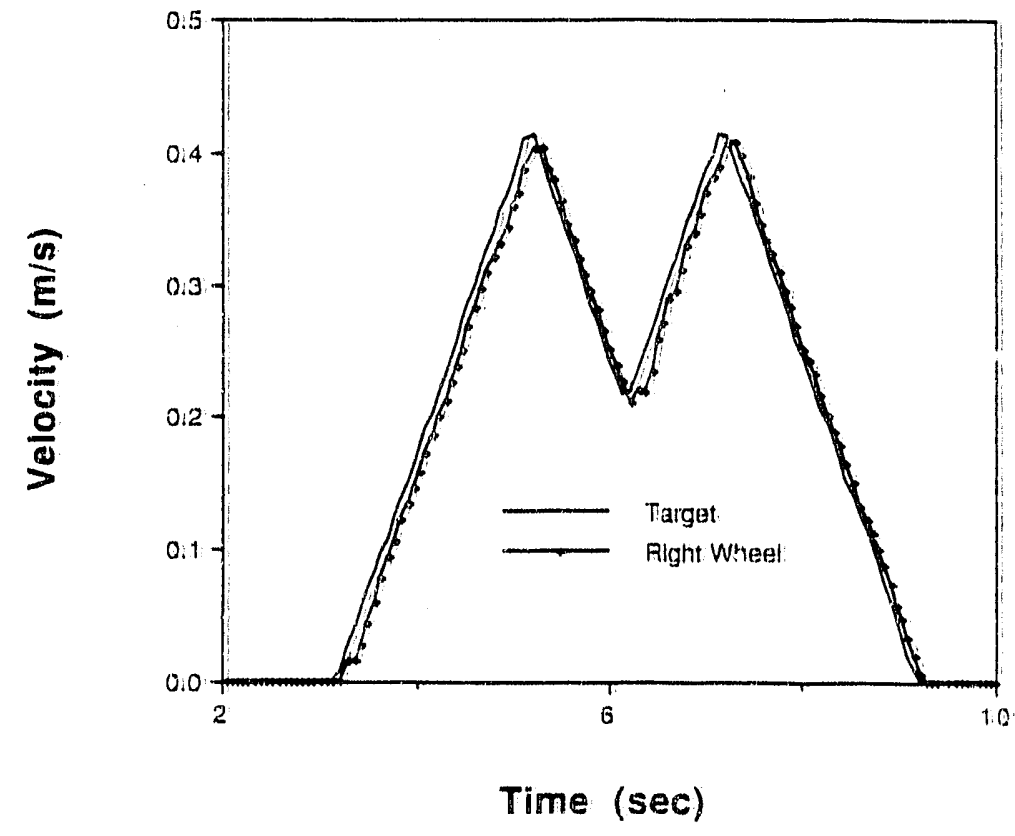

Fig. 22. Measured velocity profile for the HERMIES-III robot as it follows a bang-bang velocity target. 


\section{CONCLUSIONS}

Our objective has been to plan time-optimal motions for a mobile robot with two independently-driven: non-steerable wheels from one static configuration to the next in an unobstructed planar environment. We have used Pontryagin's Maximum Principle to prove that, when the maximum acceleration on each wheel is bounded; all optimall trajectories are bang-bang. Using this result, we have proposed a parameterization of the bang-bang trajectories which allows us to span the space of trajectories and to show that any position in the plane can be reached by a path with three switch times while any configuration can be reached by a path with four switch times. With these results, we have identified two uniquely defined subsets of paths as candidates for time-optimality.

We hare then followed a numerical procedure to verify that the paths in these subsets are the time-optimal ones: we use the unique two parameters defining. each path to calculate the switch times for that path, numerically integrate the control trajectories to calculate the Cartesian paths and some auxilary variables, use the auxilary variables to calculate the initial conditions for the dual variables, numerically integrate to calculate the dual variables, and verify that the Cartesian paths are consistent with the bang-bang. controls as required by the maximum principle. We then numerically showed that the paths in the subsets are timeoptimal by comparing them with other possible paths with larger numbers of switch. times. Being numerical, the procedure requsred an extensive examination of many cases; however, it has allowed us to precisely identify and parameterize the set of trajectories and associated controls that allow a robot to reach any configuration in the plane in a time-optimal fashion, a problem which had eluded many efforts to date.

With the set of time-optimal trajectories identified and parameterized, we have then discussed how the results can be utilized in conjunction with a simple search technique to implement the time-optimal controls on a mobile robot. For an illustrative value of $0.5 \mathrm{~m} / \mathrm{sec}^{2}$ for the bound on acceleration, comparative exa.t. ples indicate that the time-optimal paths carı be up to $30 \%$ faster than rotate-translaterotate paths that use bang-bang controls. 


\section{REFFRENCES}

1. A. Ailon and G. Langholtz, "On the Existence of Time-Optimal Control of M Mechanical Manipulators, Journal of Optimization Theary and Applications 46(1), 1-21 (1985):

2. C. S. Andersen et al., "Navigation Using Range Images on a Mobile Robot," accepted for publication in Robotics and Autonomous Systems (1992).

3. R. C. Arkin, "Integrating Behavioral, Perceptual, and World Knowledge in Reactive Navigation," Robotics and Autonomous Systems 6(1 \& 2), 105-122 (1990).

4. G. L. Blaisdell, "Performance of an Omnidirectional Wheel on Snow and Ice," Naval Engineery Journal 103(1), 34-41 (1991).

5. J. E. Bobrow, "Optimal Robot Path Planning Using the Minimum-Time Criterion," IEEE J. of Robotics and Automation 4(4), 443-450 (1988).

6. R. A. Brooks, "Elephants Don't Play Chess," Roboticy and A utonomous Systems 6(1 \& 2), 3-15 (1990).

7. Y. Chen and A. A. Desrochers, "A Proof of the Structure of the Minimum-Time Control Law of Robotic Manipulators Using a Hamiltonian Formulation," IEEE Transactions on Roboticy and Automation 6(3), 388-393 (1990).

8. A. M. Formal'skii and S. N. Osipor, "On the Problem of the Time-Optimal Manipulator Arm Turning," IEEE Transactions on Automatic Control 35(6), $714-719$ (1990).

9. H. P. Geering et al., "Time-Optimal Motions of Robots in Assembly Tasks," IEEE Transactions on Automatic Control 31(6), 512-518 (1986).

10. G. Giralt, R. Chatila, and M. Vaisset, "An Integrated Navigation and Motion Control System for Autonomous Multisensory Mobile Robois," Robotics Research: The First International Symposium, M. Brady and R. P. Paul, eds., MIT' Press, Cambridge, Massachusetts, 1984.

11. P. Jacobs, J. P. Laumond, and A. Rege, "Non-Holonomic Motion Planning for Hilare-Like Mobile Robots," Proc. of the Internationa! Symposium on Intelligent Robotics, 338-347 (1991).

12. M. E. Kahn and B. Roth, "The Near-Minimurn-Time Control of OpenLoop Articulated Kinematic Chains," ASME Journal of Dynamic Systems, Measurement, and Control 93(3), 164-172 (1971).

13. Y. Kanayama and B. I. Hartman, "Smooth Loc al Path Planning for Autonomous Vehicles," Proc. IEEE International Conference on Robotics and A utomation, 1265-1270 (1989).

14. S. M. Killough and F. G. Pin, "A Fully Omnidirectional Wheeled Assembly for Robotic Vehicles," Trans. Am. Nucl. Soc. 61 425-426 (1990). 
15. B. K. Kim and K. G. Shin, "Suboptimal Control of Industrial Manipulators with a Weighted Minimum Time-Fuel Criterion," IEEE Transactions on Automatic Control 30(1), 1-10 (1985).

16. Y. Koren and J. Borenstein, "Potential Field Methods and Their Inherent Limitations for Mobile Robot Navigation," Proc. IEEE International Conference on Roboticy and Automation, 1398-1404 (1991).

17. Y. Nakamura and H. Hanafisa, "Optimal Redundancy Control of Robot, Manipulators," Int. J. Robotics Research 6(1), 32-42 (1987).

18. M. Niv and D. M. Auslander, "Optirnal Control of a Robot with Obstacles," Proc. of the American Control Conference, 280-287 (1984).

19. S. N. Osipor and A. M. Formal'skii, "The Problem of the Time-Dptimal Turning of a Manipulator," Applied Mathematicy and Mechanics 52(6), 725-731 (1988).

20. L. S. Pontryagin et al., L. S. Pontryagin Selected Works, Volume 4: The Mathematical Theory of Optimal Procegyey, Gordon and Breach Science Publishers, New York (1986).

21. W. H. Press et al., Numerical Recipes in C: The Art of Scientific Computing, Cambridge, UK: Cambridge University Press, 1988.

22. D. B. Reister, "A New Wheel Control System for the Omnidirectional HERMIES-III Robot," accepted for publication in Robotica (1992).

23. L. G. Van Willigenburg, "First Order Controllability and the Time Optimal Control Problem for Rigid Articulated Arm Robots with Friction," Int. J. Control 51(6), 1159-1171 (1990).

24. H. A. Vasseur, F. G. Pin, and J. R. Taylor, "Navigation of a Car-Like Mobile Robot Using a Decomposition of the Environment in Convex Cells," Proc. IEEE International Conference on Robotics and Automation, 1496-1502 (1991).

25. A. Weinreb and A. E. Bryson, "Optimal Control of Systems with Hard Control Bounds," IEEE Transactions on Automatic Contral 30(11), 1135-1138 (1985).

26. C. R. Weisbin, G. de Saussure, J. R. Einctein, F. G. Pin, and E. Heer, "Autonomous Mobile Robot Navigation and Learning," Computer 22(6), 29-35 (June 1989).

27. J. Wen, "On Minimum Time Control for Robotic Manipulators," Recent Trends in Robotics: Modeling, Control, and Education, North-Holland, New York, 283-292 (1986).

28. M. Yamamoto and A. Mohri, "Planning of Quasi-Minimum Time Trajectories for Robot Manipulators (Generation of a Bang-Bang Control)," Robotica 7 , $43-47$ (1989). 


\section{INTERNAL DISTRIBUTION}

1. B. R. Appleton

2. J. E. Baker

3. A. L. Bangs

4. M. Beckerman

5. B. L. Burks

6. R. J. Carter

7. J. R. Einstein

8. C. W. Glover

9. X. Guan

10. W. R. Hamel

11. J. N. Herndon

12. J. F. Jansen

13. J. P. Jones

14. I. E. Knee

15. R. L. Kress

16-20. R. C. Mann

21. E. M. Oblow
22-26. F. G. Pin

27-31. D. B. Reister

32. J. C. Schryver

33. P. F. Spelt

34. F. J. Sweeney

35. E. C. Uberbacher

36. M. A. Unseren

37-41. R. C. Ward

42. EPMD Reports Office

43-44. Laboratory Records Department

45. Laboratory Records, ORNL-RC

46. Documcnt Reference Section

47. Central Research Library

48. ORNL Patent Section

\section{EXTERNAL DISTRIBUTION}

49. Dr. Peter Allen, Department of Computer Science, 450 Computer Science, Columbia University, New York, NY 10027

50. Mr. Harry Alter, Division of Advanced Technology Development, U.S. Department of Energy, Washington, DC 20545

51. Dr. John Baillieul, Aerospace and Mechanical Engineering Department, Boston University, 110 Cummington St., Boston, MA 02215

52. Dr. Wayne Book, Department of Mechanical Engineering, J. S. Coon Building, Room 306, Georgia Institute of Technology, Atlanta, GA 30332

53. Dr. Johann Borenstein, The University of Michigan, 1101 Beal Ave., Ann Arbor, MI 48109-2110

54. Professor Roger W. Brockett, Wang Professor of Electrical Engineering and Computer Science, Division of Applied Sciences, Harvard University, Cambridge, MA 02138

55. Professor Carl Crane, 202 Nuclear Science Center, University of Florida, Gainesville, FL 32611

56. Professor John J. Dorning, Department of Nuclear Engineering and Physics, Thornton Hall, McCormick Rd., University of Virginia, Charlottesville, VA 22901

57. Dr. Steven Dubowsky, Massachusetts Institute of Technology, Building 3, Floom 469A, 77 Massachusetts Ave., Cambridge, MA 02139

58. Dr. Ralph C. Gonzalez, Department of Electrical and Computer Engineering, The University of Tennessee, Knoxville, TN 37996-2100

59. Dr. Avi Kak, Department of Electrical Engineering, Purdue University, Northwestern Ave., Engineering Mail, Lafayette, IN 47907

60. Professor Takeo Kanade, Computer Science and Robotics, Carnegie Mellon University, Pittsburgh, PA 15213-3890

61. Dr. Jaines E. Leiss, 13013 Chestnut Oak Dr., Gaithersburg, MD 20878 
61. Professor Suzanne M. Lenhart, Department of Mathematics, The University of Tennessee, 304 Ayres Hall, Knoxville, TN 37996-1300

62. Dr. Oscar P. Manley, Division of Engineering, Mathematical, and Geosciences, Office of Basic Energy Sciences, ER-15, U.S. Department of Energy - Germantown, Washington, DC 20545

63. Professor Neville Moray, Department of Mechanical and Industrial Engineering, University of Illinois, 1206 West Green St., Urbana, IL 61801

64. Dr. Shankar Shastry, Department of EECS, 261-M Cory Hall, University of California, Berkeley, CA 94720

65. Dr. Wes Snyder, Center for Communications and Signal Processing, North Carolina State University, P.O. Box 7914, Raleigh, NC 27695-7914

66. Professor Delbert Tesar, Department of Mechanical Engineering, 26 San Jacinto, University of Texas, Austin, TX 78712

67. Professor M. A. Trivedi, Department of Electrical and Computer Engineering, The University of Tennessee, Knoxville, TN 37996-2100

68. Professor James S. Tulenko, 202 Nuclear Science Center, University of Florida, Gainesville, FL 32611.

69. Professor David K. Wehe, Department of Nuclear Engineering, 2355 Bonisteel Blvd., The University of Michigan, Ann Arbor, MI 48109-2106

70. Professor Mary F. Wheeler, Department of Mathematical Sciences, Rice University, P.O. Box 1892, Houston, TX 77251

71. Office of Assistant Manager for Energy Research and Development, U.S. Departmient of Energy, Oak Ridge Operations Office, P.O. Box 2001, Oak Ridge, TN 37831-8600

72-81. Office of Scientific Technical Information, P.O. Box 62, Oak Ridge, TN 378.31. 

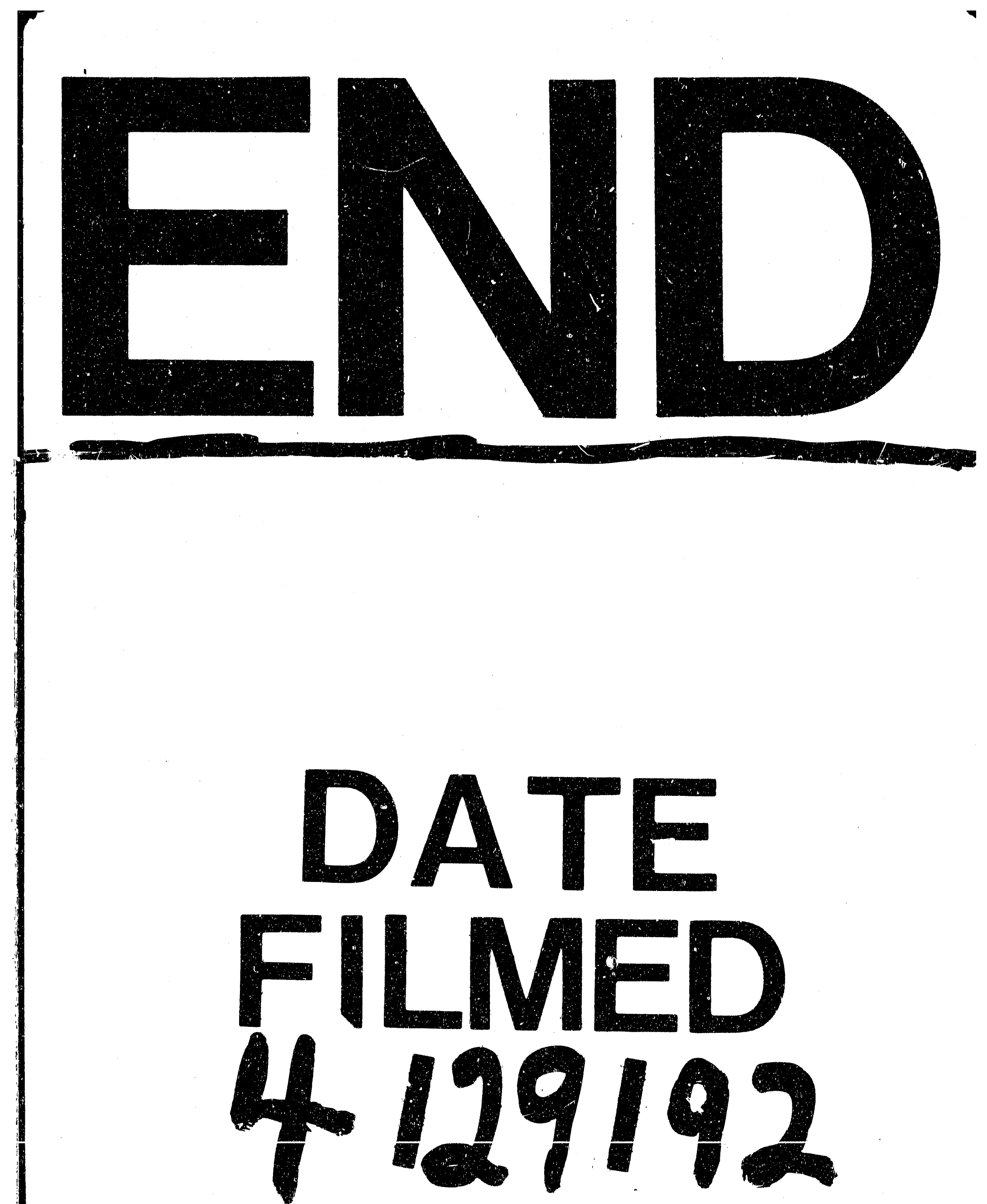


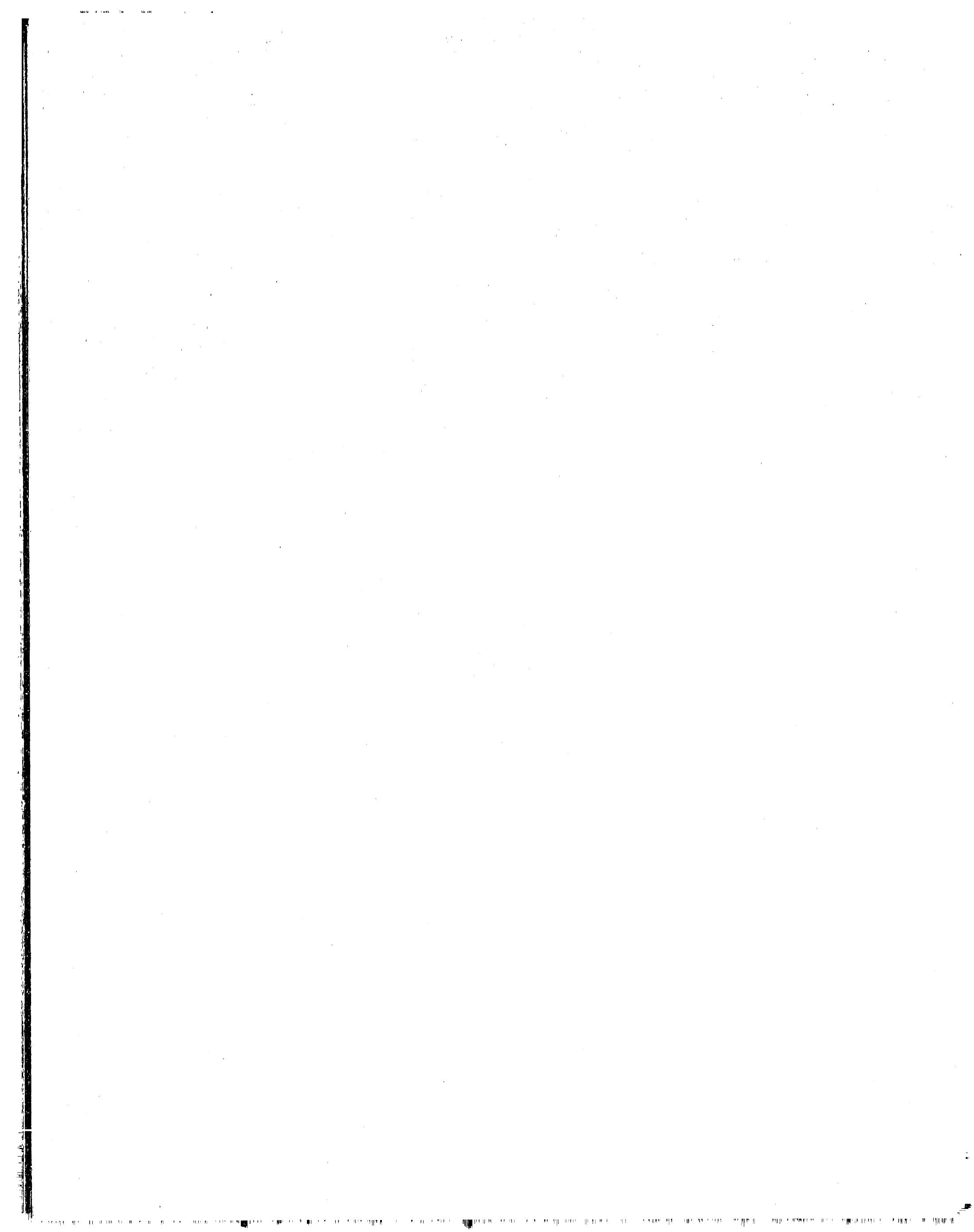

